\title{
Die Islamische Republik als Verfassungsprinzip - Ein Vergleich der Verfassungen von Afghanistan und Iran
}

\author{
Von Ramin Moschtaghi, Heidelberg ${ }^{1}$
}

\section{I. $\quad$ Einleitung ${ }^{2}$}

Der Status des islamischen Rechts hat im Verfassungsdiskurs der Staaten des Nahen und Mittleren Osten in den letzten Jahrzehnten wachsende Bedeutung erlangt. Kaum eine Frage hat im Rahmen von Verfassungsreformen oder Verfassungsneuschöpfungen in den mehrheitlich muslimischen Staaten dieser Region seit den siebziger Jahren eine solche Prominenz und öffentliche Aufmerksamkeit entfaltet wie jene der Bedeutung des islamischen Rechts für die jeweilige Rechtsordnung. Besondere Befürchtungen verbinden sich im Westen dabei mit dem Begriff einer „Islamischen Republik“; so sorgten beispielsweise Gerüchte über die Ausrufung einer „Islamische Republik Irak“ während der Verhandlungen zur neuen irakischen Verfassung nach dem Sturz des Regimes Saddam Husseins für große Beunruhigung in westlichen Staaten. Im Hinblick auf die Legitimitätswirkung, welche der Islam innerhalb konservativ islamisch geprägter Gesellschaften zu vermitteln vermag, erscheint es als nicht unwahrscheinlich, dass man in Zukunft mit weiteren islamischen Republiken in der Region rechnen muss. Es stellt sich daher die Frage, ob diese Befürchtungen auf Seiten des Westens berechtigt sind.

Diesen Befürchtungen steht ein bemerkenswerter Mangel an Literatur zu diesem Thema gegenüber. Zwar existiert eine große Anzahl von Publikationen zum islamischen Recht und seiner Vereinbarkeit oder Unvereinbarkeit mit den völkerrechtlich garantierten Menschenrechten ${ }^{3}$; Abhandlungen dazu, was aber denn eine Republik gerade zu einer islamischen macht, sind dagegen nur spärlich zu finden. Um grundsätzliche Aussagen zu dieser Frage treffen zu können und Gemeinsamkeiten der verschiedenen Verfassungsordnungen, welche

Der Verfasser ist Wissenschaftlicher Referent am Max-Planck-Institut für ausländisches öffentliches Recht und Völkerrecht, Heidelberg und Mitarbeiter im Afghanistanprojekt des Instituts, welches unter Leitung von Prof. Dr. Dr. h.c. Rüdiger Wolfrum, den Wiederaufbau der afghanischen Justiz unterstützt. Für weitere Informationen zum Projekt siehe http://www.mpil.de/ww/de/pub/ forschung/forschung_im_detail/glob_wisstransf/afghanistan_projekt.cfm. Fragen und Anregungen zu dem Artikel sind unter moschtag@mpil.de jederzeit willkommen.

Der Artikel stellt die überarbeite Version eines Vortrages mit dem Titel: „Die Islamische Republik als Verfassungsprinzip - Die Verfassungen von Afghanistan und Iran im Vergleich“ dar, welcher am 23. Juni 2007 auf der Jahrestagung des Arbeitskreises für Überseeische Verfassungsvergleichung im Reichskammergerichtsmuseum in Wetzlar gehalten wurde.

Der Begriff der Menschenrechte soll sich für die vorliegende Untersuchung auf die in der Allgemeinen Erklärung der Menschenrechten und den beiden Menschenrechtspakten statuierten Menschenrechte beschränken. 
sich für die Etablierung dieses Staatsprinzips entschieden haben, ${ }^{4}$ zu identifizieren, ist eine rechtsvergleichende Betrachtung dieser Verfassungen notwendig, wobei der Schwerpunkt der Betrachtung hier auf den Verfassungen der Islamischen Republiken (im Folgenden: I. R.) Afghanistan und Iran liegt.

Nach einer kurzen Betrachtung der Frage, inwieweit ein islamischer Staat überhaupt eine Verfassung im normativen Sinne aufweisen kann, werden zur Untersuchung, welche Auswirkungen sich aus der Einführung einer islamischen Republik für die Rechtsordnung ergeben, die aus diesem Staatsprinzip folgenden Regelungen in drei unterschiedliche Kategorien eingeteilt, welche sich aus dem Verhältnis der jeweiligen Regelungen zu den Menschenrechten ergeben. Zur ersten Kategorie werden solche Regelungen gezählt, welche sich im Hinblick auf die Menschenrechte als unproblematisch darstellen und positive Auswirkungen für deren Beachtung haben können. Zur zweiten Kategorie werden dagegen Regelungen gezählt, welche in einem eindeutigen Konflikt zu den Menschenrechten stehen. Die dritte Kategorie bilden schließlich Regelungen, welche zentral und konstitutiv für das Prinzip einer islamischen Republik sind und die zwar nicht per se im Konflikt zu den Menschenrechten stehen, die aber je nach Auslegung des islamischen Rechts zu Verstößen gegen diese führen können. Zum Vergleich der beiden Verfassungen und dem Nachweis der Ableitung der Regelungen der jeweiligen Kategorie aus dem Prinzip einer islamischen Republik werden zunächst die gemeinsamen Ursprünge der Regelungen aus dem islamischen Recht aufgezeigt, bevor ihre konkrete Ausgestaltung in den beiden untersuchten Verfassungen dargelegt wird. Anschließend an die Darstellung dieser drei Kategorien von Regelungen werden die Unterschiede zwischen den beiden untersuchten Verfassungen einer kritischen Analyse unterzogen, deren Schwerpunkt die Frage bildet, inwieweit beide Verfassungen einer von einer parlamentarischen Mehrheit getragenen Neuinterpretation des islamischen Rechts im Zeichen der Durchsetzung der Menschenrechte Raum bieten.

Dabei ist die Auswahl gerade auf diese beiden Rechtsordnungen gefallen, weil sie für einen Vergleich unter menschenrechtlicher Perspektive besonders geeignet sind. Die I. R. Iran wird international nicht nur immer wieder scharf für gravierende Menschenrechtsrechtsverstöße kritisiert, ${ }^{5}$ sie erscheint auf Grund dieser Menschenrechtsverstöße und der Ereignisse unmittelbar nach der Revolution von 1979 im Westen außerdem häufig als das abschreckende Beispiel einer islamischen Republik schlechthin. Besonders reizvoll für eine wissenschaftliche Betrachtung ist die iranische Verfassung ferner deshalb, weil die I. R. Iran weltweit die einzige islamische Republik dezidiert schiitischer Prägung darstellt. Afghanistan auf der anderen Seite gehört dagegen nicht nur zu der überwiegenden Anzahl jener islamischen Republiken, deren Mehrheitsbevölkerung sunnitischen Glaubens ist,

Zurzeit existieren mit den Islamischen Republiken Afghanistan, Iran, Mauretanien und Pakistan vier Staaten, welche sich ausdrücklich als solche bezeichnen. (letzter Besuch 01. März 2008; Amnesty International, Jahresbericht 2007, S. 189 ff.; Amnesty International, Iran - Violations of Human Rights, 1987. 
sondern in der afghanischen Verfassung kommt den Menschenrechten auch eine Rolle zu, welche unter den mehrheitlich muslimischen Staaten der Region eine Ausnahmeerscheinung darstellt. Der afghanische Staat ist nach Artikel 6 der afghanischen Verfassung ${ }^{6}$ (im Folgenden: AV) nicht nur zum Schutz der Menschenrechte und der Menschenwürde verpflichtet, sondern die Verfassung betont außerdem in Artikel 7 AV ausdrücklich die Verpflichtung des Staates die Charta der Vereinten Nationen, die internationalen Verträge und Konventionen, denen Afghanistan beigetreten ist, sowie die Allgemeine Erklärung der Menschenrechte zu achten. Hieraus ergibt sich eine besondere Dynamik der afghanischen Verfassung zwischen Islam und Menschenrechten.

\section{Der normative Charakter beider untersuchter Verfassungen}

Entscheidend für die moderne Auffassung von einer Verfassung, wie sie sich seit der zweiten Hälfte des achtzehnten Jahrhunderts entwickelt hat, ist deren normative Natur und damit ihre Verbindlichkeit für alle staatlichen Organe. ${ }^{7}$ Fraglich ist allerdings, ob diese Vorstellung überhaupt mit dem Konzept einer sich explizit als islamisch titulierenden Republik vereinbar ist, wird doch beispielsweise in Saudi-Arabien nur der Islam als Verfassung des Landes anerkannt. ${ }^{8}$ Andererseits basieren aber die Rechtsysteme der islamischen Republiken Afghanistan, Iran, Mauretanien und Pakistan alle auf geschriebenen Verfassungen. Bereits der immense Aufwand, der in beiden Staaten zur Ausarbeitung der Verfassungen betrieben wurde, spricht dafür, dass diesen nicht lediglich ein unverbindlicher Charakter zukommen sollte. In Iran kam es außerdem bereits zu Beginn des zwanzigsten Jahrhunderts vor und nach der „Konstitutionellen Revolution“ von 1906/7 zu einer hitzigen Diskussion unter den ulamā, den Gelehrten des islamischen Rechts, über die Vereinbarkeit einer verbindlichen Verfassung mit islamischem Recht. Als Ergebnis dieser Debatte befürwortete die überwiegende Mehrheit der ulamā die Idee einer normativen Verfassung als Mittel zur Begrenzung der despotischen und absolutistischen Macht des Schahs. ${ }^{9}$ Auf Grund der durch diese Debatte erfolgten Verankerung des normativen Verfassungsbegriffs im religiösen Diskurs ging man nach der Revolution von 1979 ohne große Diskussion davon aus, dass die neu zu errichtende islamische Republik auf einer geschriebenen und verbindlichen Verfassung basieren sollte. ${ }^{10}$ Die Verbindlichkeit der Verfassung wird durch

Verfassung vom 26. Januar 2004, in Kraft gesetzt durch Dekret Nr. 103, veröffentlicht im Offiziellen Anzeiger Nr. 818; deutscher Text der Verfassung unter http://www.mpil.de/shared/data/ pdf/verf_dt3.pdf (letzter Besuch 01. März 2008). Vgl. Theodor Maunz / Reinhold Zippelius, , Deutsches Staatsrecht, München, 1998, S. 29 ff.

Vgl. Silvia Tellenbach, Untersuchungen zur Verfassung der islamischen Republik Iran vom 15. Januar 1979, 1985, S. 109; Werner Ende, Religion, Politik und Literatur in Saudi-Arabien, Orient Vol. 23 (1982), S. 524 ff., 531 ff.

$9 \mathrm{Zu}$ dieser Diskussion vgl. Hairi Abdul-Hadi, Schi'ism and Constitutionalism in Iran, Leiden, 1977; Tellenbach, s. Fn 8, S. 109 ff.

10

Tellenbach, s. Fn 8, S. 109 ff. 
Artikel 72 der iranischen Verfassung ${ }^{11}$ (im Folgenden: IrV) belegt, denn dieser sieht vor, dass Gesetze nicht nur dem islamischen Recht zu entsprechen haben, sondern auch der Verfassung.

Auch in der afghanischen Verfassung finden sich Bestätigungen für ihren normativen Charakter. So hat der Oberste Gerichtshof nach Artikel 121 AV Gesetze und völkerrechtliche Verträge auf Antrag der Regierung oder eines Gerichts auf deren Vereinbarkeit mit der Verfassung hin zu überprüfen. Dies erscheint insoweit auch mit dem islamischen Recht sunnitischer Prägung vereinbar, als auch im sunnitischen Islam die Etablierung einer geschriebenen und verbindlichen Verfassung befürwortet wird, wobei man sich auf die Argumentation beruft, die Šarî'a gebe nur die Schranken für die Gemeinschaft der Muslime vor, innerhalb dieser Schranken blieben aber weite Möglichkeiten für gesetzliche Regelungen nach den Erfordernissen von Zeit und Ort. ${ }^{12}$

\section{Die Auswirkungen der Einführung einer islamischen Republik auf die Rechtsordnung}

\section{Im Hinblick auf die Verwirklichung der Menschenrechte unproblematische und positive Auswirkungen des Staatsprinzips}

Als im Hinblick auf die Verwirklichung der Menschenrechte unproblematische und positive Auswirkungen des Staatsprinzips stellen sich solche Regelungen dar, welche aus der Verpflichtung des islamischen Staates abgeleitet werden, soziale Gerechtigkeit herzustellen und die Bildung der Bevölkerung sicherzustellen.

\section{a) Die soziale Verpflichtung des islamischen Staates}

Nach Ansicht der Befürworter eines islamischen Staates besteht für diesen eine unbedingte Verpflichtung zur Herstellung sozialer Gerechtigkeit. ${ }^{13}$ Diese Verpflichtung ergibt sich aus der großen Bedeutung, welcher der sozialen Gerechtigkeit und der Brüderlichkeit, die unter Muslimen zu herrschen hat, im Islam beigemessen wird. ${ }^{14}$ Diese verpflichten den Reichen sein Gut mit dem Armen zu teilen. In diesem Zusammenhang kann auf den Koranvers

Verfassung der I. R. Iran vom 15. November 1979, mit umfassenden Änderungen vom 28. Juli 1989, Offizielle Zeitung (ruznāme je rasmi) Nr. 12957, englischer Text in: Blaustein, Albert P., (Hrsg.), Constitutions of the countries of the world, Loseblattsammlung, New York 2006, Band IX; der deutsche Text der Verfassung allerdings noch ohne die umfassenden Änderungen von 1989 findet sich bei Tellenbach, s. Fn 8, S. 59 ff.

12 Asad Muhammad, The Principles of State and Government in Islam, Berkeley, 1961, S. 1 ff., 12 ff.

13 Vgl. Asad, s. Fn 12, S. 88 ff.; Göbel, Karl-Heinrich,Moderne Schiitische Politik und Staatsidee, Opladen, 1984, S. 47, 110; siehe auch ausführlich Qutb Sayyid, Social Justice in Islam, Kairo, 2000, S. $37 \mathrm{ff}$.

14

Tellenbach, s. Fn 8, S. 186. 
51:19 verwiesen werden, welcher festlegt: „Und in ihrem Gut (war) ein Teil für den Bittenden und den verschämten Armen."15 Auch der Prophet Mohammad hat in verschiedenen von ihm überlieferten Aussprüchen die Bedeutung der sozialen Verpflichtung des Herrschers gegenüber den Untertanen betont. ${ }^{16}$ Den Vorgaben des Korans und den Überlieferungen aus dem Leben des Propheten Mohammad (Sunna) kommt im islamischen Recht insofern besondere Bedeutung zu, als sie gemeinsam als primäre Quellen des islamischen Rechts die so genannte Šarî'a bilden. Zu der Sunna des Propheten gehören die aus seinem Leben im Rahmen seiner göttlichen Mission als Prophet überlieferten und ihm zugesprochenen Aussprüche (hadith), Übungen, stillschweigenden Billigungen und Unterlassungen. Denn im Hinblick auf sein diesbezügliches Verhalten gilt Mohammad nach islamischer Lehre als unfehlbar. ${ }^{17}{ }^{18}$ Die Verpflichtung des Staates, für soziale Gerechtigkeit zu sorgen und seinen Bürgern soziale Sicherheit zu bieten, ergibt sich daher unmittelbar aus der Šarî'a. ${ }^{19}$ Schließlich wird die soziale Verpflichtung des Staates auch mit dem Gedanken begründet, dass ohne ein Minimum an materieller Ausstattung ein geistiger Fortschritt nicht möglich sei. ${ }^{20}$ Dies wird in diesem Zusammenhang allerdings immer als Fortschritt in Richtung auf den Islam hin verstanden. ${ }^{21}$

Zitiert nach der Übersetzung von Max Henning, Der Koran, Stuttgart, 2005, S. 504.

Asad, s. Fn 12, S. 87.

El Baradie Adel, Gottes-Recht und Menschen-Recht, Baden-Baden, 1983, S. 25; ausführlich zur Sunna des Propheten Birgit Krawietz, Hierarchie der Rechtsquellen im tradierten sunnitischen Islam, Berlin, 2002, S. 115 ff.; Abdal-Haqq Irshad, in: Ramadan Hisham, M. (Hrsg.), Understanding Islamic Law, Lanham, 2006, S. 4.

In Konsequenz zu den grundlegenden Unterschieden zwischen Schia und Sunna, unterscheiden sich auch die Vorstellungen über den Inhalt der Sunna zwischen der ğafari Rechtsschule und den sunnitischen Rechtsschulen erheblich. Während die Rechtschulen beider Richtungen des Islams die Sunna des Propheten als Bestandteil der Šarî'a auffassen, umfasst die für Šarî'a relevante Sunna nach dem Verständnis der ğafari Rechtsschule auch die Sunna der zwölf Imāme. Die zwölf Imāme sind Ali der Cousin und Schwiegersohn des Propheten sowie elf seiner Nachfahren aus der Ehe mit Fatima der Tochter des Propheten. Nach schiitischer Lehre sind diese die einzigen legitimen Anführer der Gläubigen und zeichnen sich durch sittlich moralische Unfehlbarkeit und eine umfassende Kenntnis der $\check{S}^{\prime} a \hat{r}^{\prime} a$ aus. Sie sind die einzigen, welche die Šarî'a authentisch zu interpretieren vermögen. Weiterführend Heinz Halm, Der schiitische Islam, München, 1994, S. 43; Hāshemi Mohammad, Hoquq e asāsiye ğomhuriye eslamiye irān (Das Verfassungsrecht der Islamischen Republik Iran), Teheran 1382 (2002), Band I Prinzipien und Grundlagen des Systems, S. 108; Harald Löschner, Die dogmatischen Grundlagen des Schiitischen Rechts, 1971, S. 86f.; Yahya Noori / Sayed Amin Hassan, Legal and Political Structure of an Islamic State, Glasgow, 1987, S. 16; Wilfried Buchta, Die iranische Schia und die islamische Einheit 1979-1996, Hamburg, 1997, S. 29; Cleave, Robert, Inevitable Doubt - Two Theories of Shī'i, Leiden, 2000, S. 1. Vgl. Asad, s. Fn 12, S. 88. 
aa) Iran

In der iranischen Verfassung nehmen Regelungen zur Ausgestaltung der sozialen Verpflichtung des islamischen Staates breiten Raum ein. Bereits in der sehr ausführlich gestalteten Präambel der Verfassung wird hinsichtlich der Wirtschaftsform des Staates ausgeführt, der islamische Staat habe die Aufgabe, ,für alle Bürger gleiche und angemessene Möglichkeiten zu sichern, Arbeit zu beschaffen und die notwendigen Bedürfnisse bei der Weiterführung des Entwicklungsprozesses zu befriedigen." Auch Artikel 3 IrV betont die diesbezüglichen Verpflichtungen des Staates. Dieser Artikel legt als eine Art von Programm eine Auflistung von sechzehn Punkten fest, deren Erfüllung dem Staat als Verfassungsauftrag zugewiesen ist. ${ }^{22}$ Artikel $3 \mathrm{Nr} .9 \mathrm{IrV}$ verpflichtet den Staat, alle ,ungerechtfertigte Bevorzugung aufzuheben und gerechte Möglichkeiten für alle auf sämtlichen materiellen und geistigen Gebieten zu schaffen“. Artikel 3 Nr. 12 IrV statuiert die Verpflichtung des Staates, die Fundamente für eine gesunde und gerechte Wirtschaft gemäß islamischen Prinzipien zu schaffen, Wohlstand herbeizuführen sowie Armut und jede Art von Entbehrung im Hinblick auf Ernährung, Wohnraum, Arbeit und Hygiene zu beseitigen. Diese eher proklamaratorischen und allgemein gehaltenen Vorgaben werden an spätere Stelle der Verfassung eingehend behandelt und teilweise präzisiert. ${ }^{23}$ So verpflichtet Artikel $43 \mathrm{IrV}$ den Staat, ,um die Armut und die Entbehrung zu beseitigen und die menschlichen Bedürfnisse im Entwicklungsprozess unter Aufrechterhaltung der menschlichen Freiheit zu befriedigen“, die Wirtschaft der Islamischen Republik Iran auf der Grundlage bestimmter Prinzipien aufzubauen. Zu diesen gehört nach Artikel 43 Nr. 1 IrV der Grundsatz, dass die Wirtschaft der Befriedigung der grundlegenden Bedürfnisse aller nach Wohnung, Nahrung, Kleidung, Hygiene, medizinischer Versorgung, Bildung und Erziehung sowie der Herstellung der notwendigen Bedingungen zur Gründung von Familien dienen muss; eine Formulierung, welche sich beinahe gleichlautend in den „Grundprinzipien einer islamischen Verfassung" findet, welche 1952 von islamischen Juristen aus Pakistan ausgearbeitet wurde. $^{24}$ Daneben wird in Artikel 43 Nr. 2 IrV die Verpflichtung des Staates zur Sicherung von Arbeitsmöglichkeiten für alle mit dem Ziel der Vollbeschäftigung statuiert.

Diese Zielbestimmungen des Staates werden teilweise durch die Einräumung subjektiver Rechte ergänzt. So verpflichtet etwa Artikel $28 \mathrm{IrV}$, welcher sich in dem Kapitel der Verfassung über die Rechte der Bürger befindet, den Staat, unter Berücksichtigung des Bedarfs der Gesellschaft Beschäftigungsmöglichkeiten für jedermann zu schaffen. Artikel 29 IrV etabliert das Recht eines jeden auf soziale Sicherung durch Versicherungen, Pensionen und ähnliche Absicherungen sowie auf Zugang zu medizinischer Versorgung. Artikel $31 \mathrm{IrV}$ begründet ein Recht jedes Staatsbürgers auf eine angemessene Wohnung. Eine

22

23

24

Vgl. auch Tellenbach, s. Fn 8, S. 132.

Vgl. Tellenbach, s. s. Fn 8, S. 132.

Maududi Sayyid Abdul A'la, Islamic Law and Constitution, Lahore, 1960, S. 337 ff., 338.; Tellenbach, s. Fn 8, S. 186. 
besondere Bedeutung kommt in der iranischen Verfassung außerdem dem Schutz und der Förderung der Familie zu. Artikel $10 \mathrm{IrV}$ stellt in diesem Zusammenhang fest, die Familie bilde ,die grundlegende Einheit der islamischen Gesellschaft“. Aus diesem Grund sieht dieser Artikel vor, dass alle Gesetze, Verordnungen und Programme des Staates auf dem Gebiet der Familienpolitik der Erleichterung der Familiengründung dienen und „zum Schutze ihrer Heiligkeit [d.h. der Familie] und zur Festigung der familiären Beziehungen auf der Grundlage des islamischen Rechts und der islamischen Ethik beruhen müssen“. Der Bezug zum Islam als Begründung für den Schutz der Familie wird hier ausdrücklich festgehalten. Die Bedeutung der Familie wird auch in der Präambel der Verfassung betont. Diese besondere Bedeutung ist auch einer der Gründe dafür, warum dem Recht auf Wohnung in der iranischen Verfassung eine hohe Relevanz zugemessen wird, ist Wohnraum doch eine wichtige Existenzvoraussetzung für Familien und erleichtert deren Gründung. ${ }^{25}$

\section{bb) Afghanistan}

Auch die afghanische Verfassung verpflichtet den Staat zur Herstellung sozialer Gerechtigkeit. Den entsprechenden Staatszielbestimmungen in der afghanischen Verfassung kommt allerdings nicht dieselbe Prominenz zu wie jenen in der iranischen. Artikel 6 AV verpflichtet den Staat zur Errichtung einer wohlhabenden und fortschrittlichen Gesellschaft, basierend auf sozialer Gerechtigkeit. Auch die Präambel der Verfassung betont den Verfassungsrang dieser staatlichen Verpflichtung, wenn sie ausführt, dass die Verfassung der Errichtung einer Zivilgesellschaft dienen soll, welche unter anderem auf sozialer Gerechtigkeit beruht. Um dem Einzelnen die Möglichkeit zu geben, diesbezüglich Forderungen an den Staat zu stellen, sieht auch die afghanische Verfassung entsprechende Individualrechte vor. Artikel 48 AV etwa bestimmt, dass jeder Afghane ein Recht auf Arbeit hat. Ebenfalls in dem Kapitel über die Grundrechte wird in Artikel 52 AV festgelegt, dass der Staat kostenlos nach Maßgabe der gesetzlichen Bestimmungen medizinische Vorsorge, ärztliche Behandlung und medizinische Einrichtungen für alle Bürger zur Verfügung zu stellen hat. Schließlich ergeben sich aus Artikel 53 AV umfangreiche Fürsorgepflichten des Staates gegenüber Kriegsversehrten, Hinterbliebenen von Kriegsopfern, Behinderten und Rentnern. Auch in Afghanistan stehen daneben nach Artikel 54 AV Familien unter besonderem Schutz des Staates. Nach dieser Vorschrift ist der Staat verpflichtet, die erforderlichen Maßnahmen zur Sicherung der körperlichen und seelischen Unversehrtheit der Familie, insbesondere von Mutter und Kind, zur Erziehung der Kinder und zur Beseitigung von Traditionen, die den Bestimmungen des Islam widersprechen, zu ergreifen. Die Formulierung der „Familie als Grundpfeiler der Gesellschaft“ in Artikel 54 AV erinnert dabei an die entsprechende Formulierung in der iranischen Verfassung. ${ }^{26}$

26 
b) Die Verpflichtung des islamischen Staates, die Bildung der Bevölkerung sicherzustellen

Der Bildung wird im Islam traditionell ein sehr hoher Wert beigemessen. Sowohl in Versen des Korans als auch in zahlreichen Überlieferungen aus dem Leben des Propheten Mohammad wird die Pflicht eines jeden Muslims betont, Wissen zu erwerben. ${ }^{27}$ So wird beispielsweise überliefert, der Prophet habe geäußert: „Wenn jemand seinen Weg auf der Suche nach Wissen geht, wird Gott seinen Weg ins Paradies einfach machen“ und „Die Suche nach Wissen ist eine heilige Pflicht für jeden Muslim“. ${ }^{28}$ Daraus wird abgeleitet, dass ein Staat, welcher seine Legitimierung auf den Islam zurückführt und die Einführung des islamischen Rechts anstrebt, jedem Bürger einen kostenlosen Zugang zu Bildung ermöglichen und für Kinder eine allgemeine Schulpflicht vorsehen muss. ${ }^{29}$

a) Iran

Aus dieser Verpflichtung erklärt sich, dass in der iranischen Verfassung Bildungsrechten und entsprechenden Staatszielbestimmungen eine besondere Bedeutung zukommt. Bereits in der programmatischen Bestimmung des Artikels 3 Nr. 3 IrV wird dem Staat aufgetragen, unentgeltliche Bildung und Erziehung sowie Leibeserziehung für alle auf sämtlichen Ebenen $\mathrm{zu}$ gewährleisten und die höhere Bildung zu fördern und für die Allgemeinheit zugänglich zu machen. Diese Verpflichtung des Staates wird in Artikel $30 \mathrm{IrV}$ noch einmal bestärkt, nach welchem der iranische Staat verpflichtet ist, die Mittel zur unentgeltlichen Bildung und Erziehung bis zum Abschluss der weiterführenden Schulen bereitzustellen und die Mittel zur Hochschulbildung, soweit es für die Bedarfsdeckung des Landes erforderlich ist, unentgeltlich anzubieten; eine Formulierung, welche teilweise an Artikel 13 Abs. 2 des Internationalen Paktes für wirtschaftliche, soziale und kulturelle Rechte (im Folgenden: IPwskR) ${ }^{30}$ erinnert. Die Verortung des Artikels $30 \mathrm{IrV}$ in dem Kapitel über die Rechte des Bürgers deutet darauf hin, dass ein individueller Anspruch auf diese Leistungen besteht.

Staat in Artikel 37 und 38 zur Herstellung sozialer Gerechtigkeit und stellt außerdem durch Artikel 35 Familien unter den besonderen Schutz des Staates. Ebenso betont die mauretanische Verfassung (Verfassung vom 16. Juli 1991, englischer Text in Blaustein, s. Fn 11, Band XII) in ihrer Präambel die Bedeutung der wirtschaftlichen und sozialen Rechte sowie der Rechte der Familie, wobei die Familie auch hier als grundlegende Einheit der islamischen Gesellschaft bezeichnet wird. Artikel 16 der mauretanischen Verfassung verpflichtet den Staat zu besonderen Schutzmaßnahmen zugunsten von Familien.

Tellenbach, s. Fn 8, S. 187; Asad, s. Fn 12, S. 86f.

28

Nach Asad, s. Fn 12, S. 86f.

29 Asad, s. Fn 12, S. 87.

30

Internationaler Pakt über wirtschaftliche, soziale und kulturelle Rechte vom 19. Dezember 1966, in Kraft getreten am 3. Januar 1976, BGBI. 1973 II S. 1569 ff., UNTS Vol. 993 S. 3. Ratifiziert durch das damalige Kaiserreich Iran am 03. Januar 1976. Afghanistan ist dem Pakt am 24. Januar 1983 beigetreten. 
bb)

Afghanistan

Auch in der afghanischen Verfassung finden sich entsprechende Bestimmungen. Artikel 17 AV begründet die Verpflichtung des Staates, die notwendigen Maßnahmen zur Förderung von Bildung und Erziehung auf allen Ebenen, zur Entwicklung der religiösen Bildung, zur Regelung und zur Verbesserung der Lage der Moscheen, der religiösen Schulen (madāris) sowie der religiösen Zentren zu ergreifen. Der Zusammenhang dieser Verpflichtung mit dem Islam und seinen Geboten wird hier deutlich sichtbar. Auch die Artikel 43 und 44 AV, welche in dem Kapitel der afghanischen Verfassung über die Rechte der Bürger zu finden sind, beschäftigen sich mit Fragen der Bildung. Artikel 43 AV gewährt allen Staatsbürgern das Recht auf Bildung und legt fest, dass der Staat bis zum Hochschulabschluss den Unterricht in den staatlichen Bildungseinrichtungen kostenlos bereitzustellen hat. Eine so weitgehende Gewährung des Rechts auf Bildung ist erstaunlich in einem wirtschaftlich eher schwachen Staat wie Afghanistan, nimmt diese Bestimmung doch das Ziel des Artikels 13 Abs. 2 b) IPwskR bereits voraus, welcher die Staaten lediglich zur allmählichen Einführung der Unentgeltlichkeit der Hochschulbildung verpflichtet. Nach Artikel 43 AV ist der Staat außerdem verpflichtet, zur gleichmäßigen Verbreitung der Bildung in ganz Afghanistan und zur Sicherung der obligatorischen mittleren Schulbildung effektive Programme zu entwickeln und umzusetzen. Entsprechende Verpflichtungen bestehen nach Artikel 44 AV im Hinblick auf die gleichmäßige Verbreitung der Bildung von Frauen, die Verbesserung der Bildung der Nomaden sowie die Beseitigung des Analphabetismus.

\section{c) Zwischenergebnis}

Es kann daher festgehalten werden, dass im Hinblick auf die Verpflichtung des islamischen Staates, soziale Gerechtigkeit zu schaffen und die Bildung seiner Bürger zu gewährleisten, die Einräumung wirtschaftlicher, sozialer und kultureller Rechten (auch) als Folge der Etablierung einer islamischen Republik erscheint. In dieser Hinsicht stehen die Auswirkungen aus diesem Staatsprinzip daher nicht nur in keinem Widerspruch zu den Menschenrechten, sondern im Gegenteil kann im Diskurs mit diesen Staaten Forderungen nach Verwirklichung wirtschaftlicher, sozialer und kultureller Menschenrechte unter Hinweis auf dieses Staatsprinzip zusätzliches Gewicht verliehen werden. Damit soll allerdings nicht etwa vertreten werden, dass die Verpflichtung zur Gewährung dieser Rechte im Diskurs mit muslimischen Staaten statt aus ihrem Charakter als universelle Menschenrechte aus dem Islam abgeleitet werden sollte. Wohl aber kann unter Hinweis auf diese Übereinstimmung Forderungen nach deren Verwirklichung zusätzliche Legitimität vermittelt werden.

Die Bedeutung der sozialen Gerechtigkeit im Islam erklärt einen Großteil der Attraktivität des Modells einer Islamischen Republik in einer Weltregion, welche von gravierender sozialer Ungleichheit, Armut und Arbeitslosigkeit geprägt ist. Nicht umsonst wird das soziale Engagement islamistischer Gruppen wie der Hamas oder den Muslimbrüdern meist als Hauptgrund für deren Popularität benannt. Für die I. R. Iran ist zu bemerken, dass dort seit der Revolution durch eine Änderung der Bildungspolitik die Alphabetisierungsquote 
der Bevölkerung gerade in den ländlichen Gebieten dramatisch erhöht werden konnte, was gerade auch den Frauen zugute kommt und sich darin zeigt, dass heute mehr als die Hälfte der Studenten Frauen sind, wohlgemerkt allerdings nur im Grundstudium. ${ }^{31}$ Nicht verschwiegen werden soll allerdings, dass gerade in der I. R. Iran die tatsächliche Umsetzung der staatlichen Verpflichtungen bezüglich der Bildungsrechte mit Diskriminierungen und gravierenden Menschenrechtsverstößen einhergeht. So sind beispielsweise die Angehörigen der Religion der Bahá'í noch immer vom Zugang zu den Hochschulen des Landes ausgeschlossen. ${ }^{32}$

\section{Im Hinblick auf die Menschenrechte problematische Auswirkungen des Staatsprinzips}

Denkt man an Regelungen des islamischen Rechts, welche unvereinbar mit den Menschenrechten sind, so tauchen als erste Assoziationen regelmäßig von diesem vorgeschriebene Strafen auf, welche wie etwa die Amputation von Gliedmaßen oder die Steinigung unvereinbar mit der Menschenwürde und dem völkerrechtlich verankerten Verbot unmenschlicher Bestrafung ${ }^{33}$ sind. Obwohl gerade die Durchsetzung dieser Strafen in der islamischen Dogmatik oft als eine der Hauptaufgaben einer islamischen Regierung gesehen wird, ${ }^{34}$ wird

Von 1998 bis 2004 wurden im Durchschnitt 57 Prozent der zu vergebenden Studienplätze an Frauen vergeben, was dazu geführt hat, dass im Jahre 2006 der Anteil weiblicher Studierender 53 Prozent betrug, im Vergleich zu 28 Prozent im Jahre 1978. Saeed Paivandi, Discrimination and Intolerance in Iran's Textbooks, Freedom House Publication, 2008, S. 10 http://www. freedomhouse.org/uploads/press_release/IranTextbookAnalysis_FINAL.pdf (letzter Besuch 05 . April 2008).

Abdelfattah Amor, Sonderberichterstatter zur Frage religiöser Intoleranz in seinem Besuchsbericht zur I. R. Iran vom 9. Februar 1996, UN Doc E/Cn.4/1996/95 Add. 2, Rn § 63; Human Rights Watch, http://www.hrw.org/english/docs/2007/09/20/iran16906.htm (letzter Besuch 05. April 2008). Problematisch mit Hinblick auf die Religionsfreiheit Andersgläubiger ist auch $\S 1$ des Gesetzes über die Ziele und Pflichten des Erziehungsministeriums (qanun e ahdäf va vazäief $e$ vezärat e ämuzesh va parwaresh) vom 25.11.1366 (1987); Offizieller Anzeiger (ruznāme ye rasmi) Nr. 12542), welcher festlegt: „...vorrangiges Ziel aller Bildung ist die Stärkung des Glaubens und der geistigen Grundlagen der Schüler, durch Aufklärung und Unterricht über die Grundlagen und Gebote des Islams sowie der wahrhaftigen zwölferschiitischen ğafari Rechtsschule..".

Vgl. zu diesem Verbot Artikel 5 der Allgemeinen Erklärung der Menschenrechte; Artikel 7 des Internationalen Pakt über bürgerliche und politische Rechte; Übereinkommen gegen Folter und andere grausame, unmenschliche oder erniedrigende Behandlung oder Strafe vom 10. Dezember 1984, in Kraft getreten am 26. Juni 1987; BGBl. 1990 II S. 246; ILM Vol. 23 S. 1027, Änderungen ILM Vol. 24 S. 535; Artikel 3 der Europäischen Konvention zum Schutze der Menschenrechte und Grundfreiheiten; Artikel 5 Abs. 2 der Amerikanischen Menschenrechtskonvention; Artikel 5 der Afrikanischen Banjul Charta der Menschenrechte und Rechte der Völker. 
dieses Problem hier nur am Rande erwähnt. Denn erstens existiert zu diesen Punkten bereits eine Menge hervorragender Literatur, welcher hier kaum etwas hinzugefügt werden könnte. ${ }^{35}$ Zweitens lässt sich, einen entsprechenden politischen Willen vorausgesetzt, die tatsächliche Anwendung dieser Strafen entweder durch Einschränkungen auf der Tatbestandsseite der entsprechenden Delikte oder durch prozessuale Hürden vermeiden, ohne dabei die Kategorien des islamischen Rechts zu verlassen. ${ }^{36}$

Häufig unbeachtet bleiben dagegen Diskriminierungen von Nicht-Muslimen, welche im islamischen Recht vorgesehen sind, dessen Durchsetzung wie noch zu zeigen sein wird, ${ }^{37}$ konstitutiv für eine islamische Republik ist. Dabei handelt es sich unter anderem um Bestimmungen, welche bestimmte Staatsämter ausschließlich Muslimen vorbehalten. So ist beispielsweise in allen Rechtsschulen des Islam anerkannt, dass das Staatsoberhaupt und alle anderen Personen, welchen die Leitung und Führung der Gläubigen obliegt, rechtgläubig sein müssen. Folglich sind alle Nicht-Muslime von der Staatsleitung ausgeschlossen. ${ }^{38}$ In der schiitischen ğafari Rechtsschule ${ }^{39}$ bezieht sich dieser Ausschluss auch auf alle nichtschiitischen Muslime, denn diese gelten nicht als rechtgläubig. ${ }^{40}$

Zur Begründung für den Ausschluss von Nicht-Muslimen wird auf die Sure 4: 59 des Korans verwiesen, welche lautet: „O ihr, die ihr glaubt, gehorchet Allah und gehorchet dem Gesandten und denen, die Befehl unter euch haben “41. Aus dieser wird geschlossen, dass nur diejenigen zur Leitung des Gemeinwesens befähigt seien, die selbst zu der angespro-

Vgl. weiterführend zu diesem Themenkomplex Baradie, s. Fn 17, 1983; sowie beispielhaft für den Fall der Aposthasie Knust Rassekh Afshar Mandana, The case of an Afghan apostate, Max Planck Yearbook of United Nations law, Vol. 10 (2006), S. $591 \mathrm{ff}$.

Vgl. hierzu An-Na'im Abdullahi Ahmed, Towards an Islamic Reformation, Civil Liberties, Human Rights, and International Law, Syracuse NY, 1990, S. 110 ff.; An-Na'im Abdullahi Ahmed, in: ders. (Hrsg.), Cross-Cultural Perspectives: A Quest for Consensus, Philadelphia, 1995, S. 34 ff.

Siehe unter 3. Für die Menschenrechte potentiell problematische Auswirkungen aus dem Staatsprinzip einer islamischen Republik.

Kadivar Mohsen, Goft o Gu je Mahnāme āftāb ba Mohsen Kadivar (Gespräche der Monatszeitschrift Āftāb mit Mohsen Kadivar), Monatszeitschrift Āftāb Nr. 27, 4. 1382 (2003); vgl. auch Tellenbach, s. Fn 8, S. 220; Reiners, Heinrich, Die klassische Islamische Staatsidee, Münster, 1968, S. 54, 135; Asad, s. Fn 12, S. 39 ff., 41; vgl. auch Māwardī, s. Fn 34, S. 12; Kurdi Adulrahman Abdulkadir, The Islamic State, London, 1984, S. 72, 92.

Der Name der Rechtsschule leitet sich von Ğafar as-Sādeq ab, der als Begründer des zwölferschiitischen Rechts gilt. Der Name Zwölferschia erklärt sich dadurch, dass die Anhänger dieser Rechtsschule des Islams an eine genealogische Linie von zwölf so genannten Imāmen glauben, welche für sie als die einzigen legitimes Anführer der muslimischen Gemeinde gelten. Die sunnitischen Kalifen werden von ihnen dagegen als Usurpatoren betrachten. Zur Schia im Detail Halm, s. Fn 18; Momen, s. Fn 40.

So der als kanonisch anerkannte schiitische Rechtsgelehrte Ibn Babuya, nach Momen Moojan, An Introduction to Schi'i Islam, New Haven, 1985, S. 158, dort auch S. 157f.; Buchta, s. Fn 18, S. 79; Kadivar, s. Fn 38; Kohlberg, Ethan, Non-Imāmi Muslims in Imāmi Fiqh, Jerusalem Studies in Arabic and Islam, Vol. 16 (1993), S. 99 ff., 99; vgl. auch Tellenbach, s. Fn 8, S. 220. 
chenen Gruppe, also den Muslimen gehörten. ${ }^{42}$ Beispiele für entsprechende Regelungen finden sich den Verfassungen aller islamischen Republiken.

a) Iran

Die iranische Rechtsordnung schließt Nicht-Muslime und auf Grund des schiitischen Charakters des Staates auch Sunniten von dem Zugang zu einer Vielzahl von Staatsämtern kategorisch aus. Um den Rahmen der vorliegenden Untersuchung nicht zu sprengen, soll hier nur das Amt des Staatspräsidenten untersucht werden.

Der iranische Staatspräsident wird gemäß Artikel $114 \mathrm{IrV}$ für eine Amtszeit von vier Jahren in allgemeiner Wahl direkt vom Volk gewählt. Nach Artikel $115 \mathrm{IrV}$ ist das passive Wahlrecht zum Amt des Staatspräsidenten von einer Reihe von Bedingungen abhängig. Insbesondere muss der Staatspräsident nach dieser Vorschrift der offiziellen Rechtsschule (mazhab) des Landes angehören und rechtgläubig (mo'men) sein. Die in Artikel $115 \mathrm{IrV}$ aufgeführten Beschränkungen des passiven Wahlrechts werden wortgleich in $\S 35$ des Gesetzes über die Wahlen zur Präsidentschaft der Islamischen Republik Iran ${ }^{43}$ wiederholt. Gemäß Artikel 12 der Verfassung ist die offizielle islamische Rechtsschule (mazhab), die schiitische ğafari Rechtsschule. Damit sind alle Staatsbürger, welche nicht dieser Rechtsschule angehören vom passiven Wahlrecht ausgeschlossen. Dies umfasst nicht nur alle Nicht-Muslime, sondern auch alle moslemischen Iraner, die nicht jener Rechtsschule angehören. Der Ausschluss der Nicht-Schiitischen Staatsbürger vom passiven Wahlrecht wird durch die in Artikel $115 \mathrm{IrV}$ zusätzlich vorgesehene Eigenschaft der Rechtgläubigkeit noch einmal betont, denn wie bereits erwähnt wurde, gelten in der ğafari Rechtsschule nur Schiiten als rechtgläubig (mo'men) ${ }^{44}$ und nach Artikel 4 IrV in Verbindung mit Artikel 12 IrV sind alle Bestimmungen der Verfassung als auf die Gebote dieser Rechtsschule basierend $\mathrm{zu}$ interpretieren.

\section{b) Afghanistan}

Auch der afghanische Staatspräsident wird nach Artikel 61 AV in allgemeiner Wahl direkt vom Volk gewählt. Artikel 62 Nr. 1 AV bestimmt, dass ein Bewerber um dieses Amt unter anderem Muslim zu sein hat. Damit ist auch in Afghanistan das Amt des Staatspräsidenten ausschließlich Muslimen vorbehalten. ${ }^{45}$

Qānun e entekhābāt e riāsat e ğamhuriye e eslāmi ye irān vom 05. 04. 1364 (1985), mağmu'e ye qavānin (Amtliche Gesetzessammlung) 1364 (1985) S. $107 \mathrm{ff}$.

44 Vgl. Fn 40.

45

Auch die pakistanische Verfassung beschränkt in Artikel 41 Abs. 2 die Wählbarkeit zum Amts des Staatspräsidenten auf Muslime, im Gegensatz zur iranischen Verfassung erfolgt dabei aber keine Festlegung auf eine bestimmte Rechtsschule des Islams. Entsprechende Bestimmungen finden sich auch in Artikel 23 der mauretanischen Verfassung. 
c)

\section{Menschenrechtliche Bewertung}

Aus der Binnenperspektive der jeweiligen Verfassungen betrachtet sind diese Ungleichbehandlungen unproblematisch, weil sowohl die iranische als auch die afghanische Verfassung zwar die Religionsausübungsfreiheit gewährleisten, nicht aber die negative Religionsfreiheit. ${ }^{46}$ In der iranischen Verfassung steht außerdem die in Artikel 20 garantierte Gleichheit der Staatsbürger ausdrücklich unter dem Vorbehalt des islamischen Rechts. Die allgemeine Gleichheitsgarantie der afghanischen Verfassung in Artikel 22 kennt zwar keinen solchen Vorbehalt. Da sich die hier aufgezeigte Ungleichbehandlung zwischen Muslimen und Nicht-Muslimen aber aus der Verfassung selbst ergibt, ist davon auszugehen, dass diese eine Ausnahme zu Artikel 22 AV darstellt.

Die auf das islamische Recht zurückzuführenden Regelungen in den beiden Verfassungen könnten allerdings gegen die völkerrechtlich geschützte negative Religionsfreiheit nicht-muslimischer Staatsbürger, gegen deren allgemeines passives Wahlrecht sowie gegen das Verbot von Diskriminierungen verstoßen. Sollte dies der Fall sein, ist zu prüfen, welcher Rang völkerrechtlichen Regelungen in den jeweiligen Verfassungsordnungen zukommt und welche Konsequenzen aus dem Verstoß gegen diese Rechte folgen.

\section{Die negative Religionsfreiheit}

Die Garantie der Religionsfreiheit ist fester Bestandteil des internationalen Menschenrechtsschutzes. Eine erste Formulierung der Religionsfreiheit als universelles Menschenrecht findet sich in Artikel 18 der Allgemeinen Erklärung der Menschenrechte ${ }^{47}$. Auch wenn die Allgemeine Erklärung der Menschenrechte als Deklaration der Generalversammlung der Vereinten Nationen formell nicht verbindlich ist, kann diese doch zumindest im Hinblick auf die in ihr verbürgte Religionsfreiheit heute als allgemeines Völkerrecht angesehen werden. ${ }^{48}$ Die Religionsfreiheit wird außerdem durch Artikel 18 des Internationalen Paktes über bürgerliche und politische Rechte (im Folgenden: IPbpR) ${ }^{49}$ sowie Artikel 14

Vgl. Artikel 12 IrV (für muslimische religiöse Minderheiten) und Artikel 13 IrV für (Christen, Juden und Zoroastrier als so genannte ,,anerkannte Minderheiten; Artikel 2 AV. Für Muslime erklärt sich die fehlende Garantie der negativen Glaubensfreiheit daraus, dass der Abfall vom Islam nach islamischem Recht verboten und strafbar ist. Zum Apostasieverbot im Islam im Detail Knust Rassekh Afshar, s. Fn 35, S. 591 ff.

Resolution 217 C (III) vom 10 Dezember 1948 U.N. Doc. A/810 (1948), S. 77

48

Frowein, Jochen Abr., Religionsfreiheit und internationaler Menschenrechtsschutz, in: Grote, Rainer / Marauhn, Thilo (Hrsg.), Religionsfreiheit zwischen individueller Selbstbestimmung, Minderheitenschutz und Staatskirchenrecht - Völker- und verfassungsrechte Perspektiven, Berlin, Heidelberg, 2001, S. 73 ff., 75.

49

Internationaler Pakt über bürgerliche und politische Rechte vom 19. Dezember 1966, in Kraft getreten am 23. März 1976, BGB1. 1973 II, S. 1534 ff., UNTS Vol. 999, S. 171 ff. 
des Übereinkommens über die Rechte des Kindes (im Folgenden: ÜRK) ${ }^{50}$ garantiert. Beide dieser Abkommen sind sowohl von Iran wie von Afghanistan unterzeichnet und ratifiziert worden, ihre Bestimmungen sind daher für beide Staaten verbindlich. ${ }^{51}{ }^{52}$ Vorbehalte gegenüber Bestimmungen dieser Verträge, welche mit islamischem Recht nicht vereinbar sind, wurden von beiden Staaten nur hinsichtlich des ÜRK eingelegt. ${ }^{53}$ Die Religionsfreiheit wird ferner in Artikel 4 des Abkommens über die Rechtsstellung der Flüchtlinge ${ }^{54}$ erwähnt. Schließlich findet sich eine Garantie der Religionsfreiheit in Artikel 1 der Erklärung über die Beseitigung aller Formen von Intoleranz und Diskriminierung aufgrund der Religion oder der Überzeugung ${ }^{55}$. $^{56}$ Die Garantie der Religionsfreiheit ist außerdem Bestandteil zahlreicher regionaler Menschenrechtsvereinbarungen. ${ }^{57}$

Konvention über die Rechte des Kindes vom 20. November 1989, 1992 II S. 121 ff., ILM Vol. 28, S. $1457 \mathrm{ff}$.

Das damalige Kaiserreich Iran hat den IPbpR ohne Vorbehalte am 4. April 1968 unterzeichnet und am 24. Juni 1975 ratifiziert. Die damalige Demokratische Republik Afghanistan ist dem Pakt am 24. Januar 1983 beigetreten.

Die I. R. Iran hat das Übereinkommen über die Rechte des Kindes am 5. September 1991 unterzeichnet und am 13. Juli 1994 ratifiziert. Afghanistan hat das Abkommen am 27. September 1990 unterzeichnet und am 28. März 1994 ratifiziert.

Die I. R. Iran ist dem ÜRK mit dem Vorbehalt beigetreten, keine Bestimmungen des Übereinkommens anzuwenden, welche unvereinbar mit islamischem Recht oder dem bereits in Kraft befindlichen Völkerrecht sind. Gegen diesen Vorbehalt haben Dänemark, Österreich und Italien Einspruch eingelegt.

Afghanistan hat bei Unterzeichnung die Erklärung abgegeben, dass sich die afghanische Regierung das Recht vorbehält, bei Ratifizierung, einen Vorbehalt hinsichtlich all jener Bestimmungen zu erklären, welche unvereinbar sind mit den Gesetzen der islamischen Šarî'a und der lokalen, effektiven Gesetzgebung, bei Ratifizierung wurde allerdings kein Vorbehalt eingelegt.

Abkommen über die Rechtsstellung der Flüchtlinge vom 28. Juli 1951, in Kraft getreten am 22. April 1954, BGBl. 1953 II S. 560 ff., UNTS Vol. 189, S. 150 ff.; Iran ist diesem Abkommen am 28. Juli 1976 beigetreten. Afghanistan ist dem Abkommen am 30. August 2005 beigetreten. Keiner der beiden Staaten legte einen Vorbehalt ein.

Der Erklärung der Generalversammlung der Vereinten Nationen über die Beseitigung aller Formen von Intoleranz und Diskriminierung aufgrund der Religion oder der Überzeugung vom 25. November 1981, UN Doc. A/Res/36/55.

Auch wenn diese Deklaration als Erklärung der Generalversammlung völkerrechtlich nicht bindend ist, hat diese doch dadurch eine nicht unerhebliche rechtliche Bedeutung, dass ihre Annahme im Konsens aller Mitgliedstaaten der Vereinten Nationen erfolgte und daher die breite Übereinstimmung der Staatengemeinschaft hinsichtlich ihres Inhalts zum Ausdruck bringt. Den Einzelgarantien dieser Erklärung kommt daher eine für die Interpretation des verbindlichen Artikels $18 \mathrm{IPbpR}$ anleitende Funktion zu. Vgl. Kimminich Otto, Religionsfreiheit als Menschenrecht, 1990, S. 139. Artikel 12 der Amerikanischen Menschenrechtskonvention; Artikel 8 der Afrikanischen Banjul Charta der Menschenrechte und Rechte der Völker. 
Der Ausschluss nicht-muslimischer Staatsbürger von der Wählbarkeit zum Amt des Staatspräsidenten stellt für diese eine Benachteiligung auf Grund ihrer Religion dar und könnte gegen ihre negative Religionsfreiheit verstoßen. Im Gegensatz zur Religionsausübungsfreiheit ist die negative Religionsfreiheit absolut garantiert und darf daher unter keinen Umständen eingeschränkt werden. ${ }^{58}$ Es widerspricht der Idee der negativen Religionsfreiheit als Freiheitsgarantie, wenn dem Einzelnen aus der Ausübung seiner geschützten Freiheit ein rechtlicher Nachteil entsteht, wie dies bei der Einschränkung seines passiven Wahlrechts auf Grund seiner Religionszugehörigkeit der Fall ist. Artikel 18 Abs. 2 IPbpR legt fest, dass niemand einem Zwang ausgesetzt werden darf, welcher seine Freiheit, eine Religion oder eine Weltanschauung seiner Wahl zu haben oder anzunehmen, beeinträchtigen würde. Der Staat darf folglich keinerlei Zwang dahingehend ausüben, einer bestimmten Religion anzugehören. ${ }^{59}$ Dabei bezieht sich der Begriff des Zwangs, wie er etwa in Artikel 18 Abs. 2 IPbpR verwendet wird, nicht nur auf das Verbot physischen Zwanges, wie er beispielsweise durch strafrechtliche Sanktionen ausgeübt werden kann, sondern schließt auch indirekte Formen von Zwang ein, wie etwa einen unzulässigen Antrieb zum Wechsel der Religion. ${ }^{60}$ Einen unzulässigen Antrieb zum Wechsel der Religion können sowohl Privilegien darstellen, welche den Anhängern eines bestimmten Bekenntnisses im öffentlichen oder privaten Recht gewährt werden, als auch als deren Kehrseite Benachteiligungen für die Anhänger eines oder mehrerer „unerwünschter“ Bekenntnisse. ${ }^{61}$ Der Staat darf deshalb weder Vor- noch Nachteile von der Zugehörigkeit zu einer bestimmten Religion abhängig machen. ${ }^{62}$ Dies wird auch durch die Ausführungen des Menschenrechtsausschusses der Vereinten Nationen in seinem General Comment zu Artikel 18 Abs. 2 IPbpR bestätigt: "Article 18.2 bars coercion that would impair the right to have or adopt a religion or belief, including the use of threat of physical force or penal sanctions to compel believers or non-believers to adhere to their religious beliefs and con-

Partsch, Karl Josef, Freedom of Conscience and Expression, and Political Freedoms, in: Henkin, L., The International Bill of Rights, S. 209 ff., 212; Nowak, Manfred, U.N. Covenant on Civil and Political Rights - CCPR Commentary, 2.Auflage 2005, S. 425; Joseph, Sarah / Schulz, Jenny / Castan, Melissa, The International Covenant on Civil and Political Rights - Cases, Materials and Commentary, 2004, S. 505; Menschenrechtsausschuss, General Comment Nr. 22 zu Artikel 18 IPbpR vom 30. Juli 1993, UN Doc. CCPR/C/21/Rev.1/Add.4 § 3; zu diesem Punkt in der Europäischen Menschenrechtskonvention vgl. auch das Urteil des EGMR Kokkinakis gegen Griechenland, Serie A 260-A. Ziffer 33; Konrad Sahlfeld, Aspekte der Religionsfreiheit, 2004, S. 98. Nowak, s. Fn 58, S. 416.

60 Vgl. Bossuyt, Marc J., Guide to the "Travaux Préparatoires" of the International Covenant on Civil and Political Rights", Dordrecht, 1987, S. 362; Nowak, s. Fn 58, S. 416; Menschenrechtsausschuss, General Comment Nr. 22, s. Fn 58, § 9. Vgl. Nowak, s. Fn 58, S. 416; Menschenrechtsausschuss, General Comment, Nr. 22 s. Fn 58, § 9. 
gregations, to recant their religion or belief or to convert. Policies or practices having the same intention or effect, such as, for example, those restricting access to education, medical care, employment or the rights guaranteed by article $25^{63}$ [zu denen unter anderem das Recht auf gleichberechtigte Wählbarkeit gehört] and other provisions of the Covenant, are similarly inconsistent with article 18.2."64 65 Die Monopolisierung politischer Ämter durch die Anhänger der Staatsreligion vermag einen indirekten Zwang auf Personen auszuüben, dieses Bekenntnis zumindest äußerlich anzunehmen, falls sie eine politische Kariere anstreben. Dabei kann es keine Rolle spielen, dass die Chancen eines einzelnen NichtMuslimen auf das Präsidentenamt in einem mehrheitlich muslimischen Staat gering sind. Auch kann das Vorliegen eines indirekten Zwanges nicht mit der Argumentation entkräftet werden, dass vom Einzelnen erwartet werden könne, einem Anreiz zum Wechsel der Religion zu widerstehen, weil bestimmte Staatsämter nur Angehörigen eines bestimmten religiösen Bekenntnisses vorbehalten sind. Wie sich aus Artikel 18 Abs. 2 IPbpR ergibt, untersagt die negative Religionsfreiheit jeglichen Zwang, welcher die Freiheit einer Person beeinträchtigt einer bestimmten Religion anzugehören, ohne dabei eine Erheblichkeitsschwelle oder ähnliches aufzustellen. Dies zeigt sich schon daran, dass in Artikel 18 Abs. 2 IPbpR das Wort „,beeinträchtigen“, impair im englischen Original, bewusst gewählt wurde, um der Vorschrift einen möglichst weiten Anwendungsbereich zu eröffnen ${ }^{66}$ und damit jede Beeinträchtigung und jeden Zwang auszuschließen. Zweitens wird Nicht-Muslimen bereits die Möglichkeit einer gleichberechtigten Teilnahme an den Präsidentenwahlen verwehrt, und für diese Benachteiligung ist ihre tatsächliche Chance, dieses Amt auszuüben irrelevant.

Das Ergebnis, dass der Ausschluss von Nicht-Muslimen von der Wählbarkeit zum Präsidentenamt gegen deren völkerrechtlich geschützte negative Religionsfreiheit verstößt, vermag auch nicht durch einen Verweis auf die Regelungen in einigen europäischen Staaten wie etwa Norwegen und Großbritannien erschüttert zu werden, wo das erbliche Amt des Monarchen und Staatsoberhaupts ausschließlich Angehörigen der Staatsreligion vorbehalten ist, ${ }^{67}$ ohne dass dies bis heute international auf Kritik gestoßen wäre. ${ }^{68}$ Erstens werden

63

64

65

66

67

68

Hervorhebung durch den Autor.

General Comment Nr. 22, s. Fn 58.

Vgl. hierzu auch das Urteil des EGMR Hoffmann gegen Österreich, Urteil Nr. 151992/360/434 vom 23. Juni 1993, Europäische Grundrechts Zeitung 1996, 648 ff., 652; vgl. zu diesem Urteil auch Frowein, s. Fn 62, S. 371; ders., s. Fn 48, S. 73 ff., 80.

Bossuyt, s. Fn 60, S. 362.

Vgl. Artikel 4 der norwegischen Verfassung vom 16. Mai 1814 in der Fassung vom 20. Februar 2006.

Vgl. hierzu auch Giegerich, Thomas, Religionsfreiheit als Gleichheitsanspruch und Gleichheitsproblem, in: Grote, Rainer / Marauhn, Thilo (Hrsg.), Religionsfreiheit zwischen individueller Selbstbestimmung, Minderheitenschutz und Staatskirchenrecht - Völker- und verfassungsrechte Perspektiven, Berlin - Heidelberg, 2001, S. 241 ff., 268. 
diese Ämter nicht durch Wahl besetzt, sondern vererbt, so dass es bereits deshalb an der Vergleichbarkeit der Position dieser Monarchen mit einem vom Volk aus seiner Mitte gewählten Staatspräsidenten fehlt. Entscheidend ist allerdings, dass den europäischen Monarchen anders als dem iranischen sowie insbesondere dem afghanischen Staatspräsidenten eine nahezu ausschließlich repräsentative Funktion zukommt. Die beiden Staatspräsidenten dagegen nehmen als Vertreter ihres Volkes direkt an der Gestaltung der öffentlichen Angelegenheiten und der Politik des Landes teil und vermögen diese maßgeblich zu beeinflussen. Auch wenn in den entsprechenden Regelungen hinsichtlich dieser Monarchen daher keine Verletzung der negativen Religionsfreiheit der Anhänger anderer Bekenntnisse als der Staatsreligion zu sehen sein sollte, was hier nicht entschieden werden muss, so fehlt es in jedem Fall an der Vergleichbarkeit ihrer Position mit der Position des Staatspräsidenten in der iranischen beziehungsweise der afghanischen Verfassung.

\section{bb) Das Diskriminierungsverbot}

Das Verbot von Diskriminierungen ist heute fester Bestandteil des internationalen Menschenrechtsschutzes. Auf einem universellen Niveau wurde das Verbot von Diskriminierungen bereits in der Charta der Vereinten Nationen ${ }^{69}$ als eines der Ziele und Prinzipien der neu zu gründenden Organisation verankert. ${ }^{70}$ Eine erste Ausformulierung des Diskriminierungsverbotes als Menschenrecht findet sich in Artikel 2 und 7 der Allgemeinen Erklärung der Menschenrechte, wobei erstere Bestimmung ein akzessorisches Diskriminierungsverbot im Hinblick auf die in der Erklärung verkündeten Rechte und Freiheiten enthält. Auch im Internationalen Pakt über bürgerliche und politische Rechte findet sich in Artikel 2 Abs. 1 IPbpR ein entsprechendes akzessorisches Verbot von Diskriminierungen sowie ein allgemeines Diskriminierungsverbot in Artikel $26 \mathrm{IPbpR}$. Akzessorische Verbote von Diskriminierungen finden sich ferner in Artikel 2 Abs. 1 ÜRK und in Artikel 2 Abs. 2 des IPwskR. $^{71}$ Ausdrücklich gegen jede Art von Diskriminierungen auf Grund der Religion

Charta der Vereinten Nationen vom 26. Juni 1945, in Kraft getreten 24. Oktober 1945, BGB1. 1973 II S. 431 ff.

Vgl. die Artikel 1 Abs. 3, 13 Abs. 1 b), 76 c) der Charta; vgl. auch ICJ Reports 1971, Gutachten vom 21. Juni 1971 zur fortgesetzten südafrikanischen Präsenz in Namibia, (Legal Consequences for States of the Continued Presence of South Africa in Namibia notwithstanding Security Council Resolution 276 (1970)), S. 57 \$ 131; Kimminich, s. Fn 56, S. 82.

Darüber hinaus existiert eine Reihe von Abkommen, welche sich gezielt gegen Diskriminierungen in bestimmten Bereichen oder gegenüber bestimmten Gruppen richten. Zu nennen sind dabei die Konvention Nr. 111 der Internationalen Arbeitsorganisation gegen Diskriminierung in Beschäftigung und Beruf (Konvention vom 25. Juni 1958, in Kraft getreten am 15. Juni 1960, BGB1. 1961 II S. 97 ff., UNTS Vol. 362 S. 31 ff.), das Internationale Übereinkommen zur Beseitigung jeder Form von Rassendiskriminierung (Übereinkommen vom 7. März 1966, in Kraft getreten am 4. Januar 1969, BGB1. 1969 II S. 961 ff., UNTS Vol. 660 S. 195 ff.), die UNESCO Konvention gegen Diskriminierung im Unterrichtswesen (Übereinkommen vom 15. Dezember 1960, BGBl. 1968 II S. 386ff.; UNTS Vol. 429 S. 93 ff.) sowie das Übereinkommen zur Beseitigung jeder 
wendet sich ferner die Erklärung der Generalversammlung der Vereinten Nationen über die Beseitigung aller Formen von Intoleranz und Diskriminierung aufgrund der Religion oder der Überzeugung ${ }^{72}$. Auch in der Erklärung der Generalversammlung über die Rechte von Personen, die nationalen oder ethnischen, religiösen und sprachlichen Minderheiten angehören $^{73}$, finden sich zahlreiche Bestimmungen in Bezug auf das Verbot von Diskriminierungen der Angehörigen von Minderheiten. Daneben sind in diesem Zusammenhang auch regionale Menschenrechtsinstrumente zu nennen, welche ein Verbot von Diskriminierungen im Hinblick auf die dort garantierten Rechte beinhalten, wie etwa die Europäische Konvention zum Schutze der Menschenrechte und Grundfreiheiten ${ }^{74}$, die Amerikanische Menschenrechtskonvention ${ }^{75}$ und die afrikanische Banjul Charta der Menschenrechte und Rechte der Völker ${ }^{76}$.

Eine Diskriminierung liegt vor bei einer Ungleichbehandlung, welche auf gruppenbezogene Merkmale, wie sie beispielsweise in Artikel 2 Abs. 1 IPbpR genannt sind, gestützt wird, wenn diese ungerechtfertigt ist und den Betroffenen durch Behinderung oder Vereitlung der Ausübung gewisser Rechte einen (Rechts-) Nachteil auferlegt. ${ }^{77}$ Zu beachten ist, dass die Hürden, welche eine Ungleichbehandlung zu ihrer Rechtfertigung zu überwinden hat, umso höher sind, je eher diese auf einem der beispielsweise in den Artikeln 2 Abs. 1 und $26 \mathrm{IPbpR}$ namentlich genannten und für Diskriminierung besonders anfälligen Kriterium beruht. ${ }^{78}$ Eine Ungleichbehandlung auf Grund eines der unter anderem in Artikel 2

Form von Diskriminierung der Frau (Übereinkommen vom 18. Dezember 1979, in Kraft getreten 3. September 1981, BGBl. 1985 II S. 647 ff., ILM Vol. 19 S. 34 ff.).

vgl. zu dieser Fn 56.

73

74

Erklärung über die Rechte von Personen, die nationalen oder ethnischen, religiösen und sprachlichen Minderheiten angehören vom 18. Dezember 1992, UN Doc. A/47/135.

Artikel 14 Konvention zum Schutze der Menschenrechte und Grundfreiheiten vom 4. November 1950, für Deutschland in Kraft seit dem 03. September 1953 geändert durch Protokoll Nr. 11 vom 11. Mai 1994; BGBl. 1952 II S. 685ff. sowie insbesondere das Protokoll Nr. 12 zur Konvention zum Schutze der Menschenrechte und Grundfreiheiten vom 04. November das sich speziell mit diesem Thema befasst.

Artikel 1 Abs. 1 der Amerikanischen Menschenrechts Konvention vom 22. November 1969, in Kraft seit 18. Juli 1978, UNTS Vol. 1144 S. 123 ff.

Artikel 2 der Banjul Charta der Menschenrechte und der Rechte der Völker, vom 27. Juni 1981, in Kraft seit 21. Oktober 1986, ILM Vol 21 S. 58.

König, Doris / Peters, Anne, in: Grote, Rainer / Marauhn, Thilo (Hrsg.), EMRK/GG Konkordanzkommentar, Tübingen, 2006, S. 1142; Menschenrechtsausschuss, General Comment Nr. 18 vom 10. November 1989, § 6f.; Nowak, s. Fn 58, S. 46; Joseph / Schulz / Castan, s. Fn 58, S. 680.

Vgl. Tomuschat, Christian, Equality and Non-Discrimination under the International Covenant on Civil and Political Rights, in: von Münch, Ingo, Staatsrecht-Völkerrecht-Europarecht, Festschrift für Hans-Jürgen Schlochauer, Berlin, 1981, S. 691 ff., 713; Menschenrechtsausschuss, Einzelbeschwerde Nr. 919/2000, Müller und Engelhard gegen Namibia vom 26. März 2002, § 6.7, UN Doc. A/57/40 (Vol. II), in: Annual Report of the Human Rights Committee 2002, S. 243 ff., 250; Tufyal Choudhury, Interpreting the Right to Equality Under Article 26 of the International Cove- 
Abs. $1 \mathrm{IPbpR}$ genannten für Diskriminierungen besonders anfälligen, gruppenspezifischen Merkmale, wie der Religion, kann daher nur dann gerechtfertigt sein, wenn sie sich auf vernünftige und objektive Gründe stützt. ${ }^{79}$ Dies ist der Fall wenn die unterschiedliche Behandlung ein legitimes Ziel verfolgt und eine Proportionalität zwischen den angewandten Mitteln und dem zu verfolgten Ziel besteht. ${ }^{80}$

Vergleichsgruppen, zwischen denen eine Ungleichbehandlung auf Grund ihres religiösen Bekenntnisses stattfindet, sind muslimische und nicht-muslimische Staatsbürger. Der Rechtsnachteil liegt hier im Ausschluss letzterer von der Wählbarkeit zum Amt des Staatspräsidenten. Betroffenes Recht ist das unter anderem von Artikel 25 b) IPbpR geschützte Recht und die Möglichkeit jedes Staatsbürgers, bei allgemeinen Wahlen gewählt werden zu können. Zwar wird in Artikel 25 b) IPbpR selbst nicht bestimmt, welche Staatsorgane im Einzelnen durch Wahlen zu besetzen sind, vielmehr bleibt diese Entscheidung grundsätzlich den Vertragsstaaten und ihren jeweiligen Verfassungsmodellen überlassen. ${ }^{81}$ In jedem Fall aber gilt, dass wann immer nach der nationalen Verfassungsordnung der Vertragsstaaten ein bestimmtes staatliches Organ, welches Hoheitsaufgaben erfüllt, durch Wahl zu besetzen ist, die in Artikel 25 b) IPbpR statuierten Wahlrechtsgrundsätze zu beachten sind. ${ }^{82}$ Dies gilt folglich auch für die Wahl des Staatspräsidenten sowohl in der I. R. Iran als auch in der I. R. Afghanistan, da beide Vertragsstaaten des IPbpR sind. Dabei ist es für eine diskriminierungsrelevante Benachteiligung nicht erforderlich, dass Artikel 25 b) IPbpR selbst verletzt ist, eine bloße Betroffenheit des Rechts reicht vielmehr aus. ${ }^{83}$ Eine

nant on Civil and Political Rights, European Human Rights Law Review, Vol 8 (2003), S. 24 ff., 44.

Nowak, s. Fn 58, S. 46; vgl. auch Peukert, Wolfgang, in: Frowein, Jochen Abr. / Peukert, Wolfgang, Europäische Menschenrechtskonvention, Kehl, 1996, S. 453; König / Peters, s. Fn 77, S. 1125; 1204 ff.; Menschenrechtsausschuss, General Comment Nr. 18, s. Fn 77, § 13; Brinkmeier, Friedericke, Der allgemeine völkerrechtliche Diskriminierungsschutz, insbesondere nach Art. 26 des Internationalen Paktes über bürgerliche und politische Rechte, in: Klein, Eckart (Hrsg.), Rassische Diskriminierung - Erscheinungsformen und Bekämpfungsmöglichkeiten, Berlin, 2002, S. 81 ff., 105; Menschenrechtskommission, Einzelbeschwerde Nr. 1306/2004 Erlingur Sveinn Haraldsson und Örn Snævar Sveinsson gegen Island, vom 14. Dezember 2007, CCPR/C/91/D/1306/2004, § 10.2; EGMR Belgischer Sprachenfall, Entscheidung vom 23. Juli 1968, Series A, Vol. 6, § 10 S. 34; ders., Fall Inze, Entscheidung vom 28. Oktober 1987, Series A, Vol. 126, § 41 S. 18; ders., McMichael gegen Vereinigtes Königreich, Entscheidung vom 24. Februar 1995, Series A, Vol. 126, B § 97 S. 58.

Nowak, s. Fn 58, S. 46; EGMR, Fn 79; König / Peters, s. Fn 77, S. 1125.

Nowak, s. Fn 58, S. 574; Bossuyt, s. Fn 60, S. 474 f.

82

Nowak, s. Fn 58, S. 574; Menschenrechtsausschuss, General Comment No. 25 zu Art. 25 IPbpR vom 12. Juli 1996, UN Doc. CCPR/C/21/Rev.1/Add.7 § 21.

83

Vgl. Opsahl Torkel, Equality in Human Rights Law-With Particular Reference to Article 26 of the ICCPR, in: Nowak, Manfred / Steurer, Dorothea / Tretter, Hanens (Hrsg.), Fortschritt im Bewusstsein der Grund- und Menschenrechte - Festschrift für Ermacora, Kehl, 1988, S. 51 ff., 54; Nowak, s. Fn 58, S. 36. 
solche ist hier durch den Ausschluss von nicht-muslimischen Staatsbürgern von der Wählbarkeit gegeben.

Teilweise wird eine Diskriminierung im Hinblick auf den Ausschluss von Nicht-Muslimen von den obersten Staatsämtern in islamischen Ländern bereits deshalb verneint, weil deren Chancen, in ein solches Amt gewählt zu werden, durch das Spiel der ,politischen und emotionalen“" Kräfte so gering seien, dass der Staatspräsident ohnehin dem Bekenntnis der überwältigenden Mehrheit der Bevölkerung angehören würde. ${ }^{84}$ Diese Argumentation kann aus mehreren Gründen nicht überzeugen. Erstens kommt einem per Gesetz verordneten Ausschluss von Nicht-Muslimen von den obersten Staatsämtern eine nicht zu unterschätzende Symbolwirkung zu. ${ }^{85}$ Denn damit wird diesen nicht nur das Gefühl vermittelt, keinen vollwertigen Teil der Gesellschaft darzustellen, sondern es verfestigt sich auch der Eindruck, der Status als Nicht-Muslim stelle etwas Negatives dar, das ein weniger an Rechten nach sich zieht. Außerdem ist es unzulässig, von der politischen Konstellation in einem Staat oder aus den traditionellen Gegebenheiten einer Gesellschaft heraus auf das Nichtvorliegen einer Diskriminierung zu schließen. Wie der Menschenrechtsausschuss der Vereinten Nationen festgestellt hat, können Traditionen und Befindlichkeiten der Bevölkerung keine Rechtfertigung für Diskriminierungen darstellen, ${ }^{86}$ ist es doch gerade auch das Ziel des völkerrechtlichen Diskriminierungsverbotes, überlieferte diskriminierende Praktiken für Gegenwart und Zukunft zu unterbinden. Auch wenn es beispielweise noch vor wenigen Jahrzehnten unvorstellbar gewesen wäre, dass ein Afroamerikaner eine realistische Aussicht auf das Amt des Präsidenten der Vereinigten Staaten von Amerika hat, so ist es doch offensichtlich, dass deren Ausschluss vom passiven Wahlrecht auch zu dieser Zeit bereits eine Diskriminierung dargestellt hätte. Schließlich wird bei einer derartigen Argumentation übersehen, dass es sich bei dem von Artikel 25 b) IPbpR garantierten Wahlrecht nicht um das Recht handelt, ein durch Wahl zu besetzendes Amt zu bekleiden und allenfalls hinsichtlich eines solchen Rechts könnten die Erfolgsaussichten eines Bewerbers überhaupt eine Rolle spielen. Vielmehr handelt es sich bei diesem um ein Recht auf Partizipation, das folglich bereits dann verletzt ist, wenn die Teilnahme eines Kandidaten an der Wahl verhindert wird, egal welche Aussicht ein Bewerber darauf hat, tatsächlich ein bestimmtes Amt zu besetzen. Der Schutzbereich des Diskriminierungsverbots ist folglich beim Ausschluss nicht-muslimischer Präsidentschaftskandidaten in jedem Fall eröffnet.

In der iranischen rechtswissenschaftlichen Literatur und in den Schriften der Befürworter eines islamischen Staates wird versucht die unterschiedliche Behandlung von Muslimen und Nicht-Muslimen beziehungsweise in der I. R. Iran auch von Sunniten und Schiiten

Amor Abdelfattah, Verfassung und Religion in den muslimischen Staaten, Gewissen und Freiheit Nr. 50 (1998), S. 117 ff., 134.; Asad, s. Fn 12, S. 40.

Giegerich, s. Fn 68, S. 241 ff, 268.

86

Vgl. Menschenrechtsausschuss, Einzelbeschwerde Nr. 919/2000, s. Fn 78, S. 250, § 6.8; ebenso EGMR, Burghartz gegen Schweiz, Beschwerde Nr. 16213/90, Urteil vom 22 Februar 1994, dort Rn 28. 
durch die Argumentation zu rechtfertigen, dem Staatspräsidenten obliege die politische Leitung eines Staatswesens, dessen Bevölkerungsmehrheit sich aus Staatsbürgern muslimischen Glaubens zusammensetze und dessen offizielle Staatsreligion der Islam, beziehungsweise im Falle Irans dessen ğafaritische Rechtsschule, sei. Aus diesem Grunde müsse auch der Staatspräsident der Staatsreligion angehören. ${ }^{87}$ Auch stelle die verfassungsmäßige Ordnung des islamischen Staates ein politisches System dar, welches fest auf den Glauben an den Islam und dessen Gebote, als Ideologie des Staates, beruhe. Es sei daher selbstverständlich, dass insbesondere diejenigen Personen, welche oberste Staatsämter bekleiden und politische Grundsatzentscheidungen zu treffen hätten, von diesem Glauben erfüllt und überzeugt sein müssten, um die Beständigkeit des Systems zu gewährleisten. Von NichtMuslimen könne dagegen nicht erwartet werden, dass sie die ideologischen Ziele des Islams teilen. ${ }^{88}$ Interessanterweise wird dabei auf den Kommunismus verwiesen und angeführt auch in einem kommunistischen Staatssystem sei es selbstverständlich gewesen, dass ein Nicht-Kommunist nicht Staatsoberhaupt sein könne. ${ }^{89}$ Als Beleg für diese Einschätzung wird in der iranischen Literatur ferner die Formel des obligatorischen Amtseides des Staatspräsidenten herangezogen. Dieser verpflichtet den Staatspräsident, nicht nur das System der Islamischen Republik zu schützen, sondern auch den Glauben ,,an den Propheten und die reinen Imāme zu verbreiten“. 90

Eine solche Begründung kann nach den völkerrechtlichen Vorgaben keine Rechtfertigung für eine unterschiedliche Behandlung auf Grund der Religion darstellen. Soweit alleine darauf abgestellt wird, dass Nicht-Muslime nicht zum Mehrheitsbekenntnis der Bevölkerung gehören, fehlt es bereits an einem legitimen Zweck für die Ungleichbehandlung. Sinn und Zweck des völkerrechtlichen Diskriminierungsverbotes ist es gerade, Unterscheidungen anhand der beispielsweise in Artikels 2 Abs. 1 IPbpR aufgezählten, für Diskriminierungen besonders anfälligen Kriterien bei der Gewährleistung der Menschenrechte zu verhindern. ${ }^{91}$ Das völkerrechtliche Diskriminierungsverbot verpflichten die Vertragsstaaten folglich dazu, ihre Rechtssysteme dahingehend anzupassen, dass Unterschiede der Rasse $^{92}$, der Sprache oder eben der Religion nicht als Rechtfertigungen dafür dienen kön-

Asad, s. Fn 12, S. 41; vgl. auch Hāshemi, s. Fn 87, S. 269 f.; vgl. auch die Aussage des iranischen Außenministers in Besuchsbericht Amor, s. Fn 32, Rn 17.

Das Kriterium der Rasse ist außerordentlich problematisch und entbehrt jeglicher wissenschaftlicher Erkenntnis und lässt sich allenfalls auf die Hautfarbe von Personen beziehen. Dahm, Georg / Delbrück, Jost / Wolfrum, Rüdiger, Völkerrecht, Band I/2, Berlin, 2002, S. 280; ausführlich zu dem Begriff der Rasse und seiner Problematik, Makkonen, Timo, Identity, Difference and Otherness, Helsinki, 2000, S. 20 ff. 
nen, der gleichberechtigten Ausübung von Menschenrechten Hindernisse zu bereiten. ${ }^{93}$ Alleine auf das religiöse Bekenntnis eines Kandidaten und den Verweis auf die Religion der Bevölkerungsmehrheit lässt sich ein Ausschluss vom passiven Wahlrecht daher nicht stützen. ${ }^{94}$ Dies wurde vom Europäischen Gerichtshof für Menschenrechte in seiner Entscheidung im Falle Hoffmann gegen Österreich unzweifelhaft klargestellt. ${ }^{95}$ Auch wenn weder die I. R. Iran noch die I. R. Afghanistan Vertragsparteien der Europäischen Menschenrechtskonvention sind, so bietet die Rechtsprechung des Europäischen Gerichtshofes für Menschenrechte doch einzigartige Beispiele für eine Konkretisierung der Religionsund Weltanschauungsfreiheit im Einzelnen. Die Grundsätze, welche für die auch in Europa noch sehr heterogene Situation des Verhältnisses von Staat und Religion entwickelt wurden, können deshalb auch international als Beispiele von erheblicher Bedeutung sein. ${ }^{96}$

Bei der Absicht, die Beständigkeit der verfassungsmäßigen Ordnung zu garantieren, handelt es sich zwar grundsätzlich um ein vernünftiges und damit legitimes Ziel, entspricht es doch der international üblichen Praxis, die Amtsträger des Staates auf ihre Verfassungstreue zu verpflichten. ${ }^{97}$ Auch hier liegt die Problematik der Argumentation aber darin, dass Religionszugehörigkeit und Verfassungstreue gleichgesetzt werden. Es handelt sich daher auch bei diesem Argument letztlich um eine Begründung, welche die Ungleichbehandlung alleine auf einen Unterschied in der Religion stützt. Auch wenn das Völkerrecht dadurch, dass es die Einrichtung einer Staatsreligion akzeptiert, mittelbar zum Ausdruck bringt, dass es die Gleichheit der Religionsgemeinschaften nicht garantiert, darf sich aus diesem privilegierten Status der Gemeinschaft keine Diskriminierung des einzelnen Anhängers einer anderen Religion entwickeln. ${ }^{98}$ Die Religionsausübungsfreiheit der muslimischen Bevölkerungsmehrheit kann diesen keinen Anspruch auf ein Monopol auf Staatsämter gewähren. Es ist mit dem völkerrechtlichen Diskriminierungsverbot nicht zu vereinbaren, wenn Anhänger bestimmter Religionen oder Weltanschauungen ohne Ansehen der Person generell für unzuverlässig und für nicht geeignet zur Gewährleistung der Aufrechterhaltung der verfassungsmäßigen Ordnung erklärt werden, ohne dass eine Einzelfallprüfung erfolgt. ${ }^{99}$ Da es

Capotorti, Francesco, Study on the Rights of Persons belonging to Ethnic, Religious and Linguistic Minorities, United Nations Study Series 5, 1991, UN Publication Sales No E.91.XIV.2, S. 40 f.; Thornberry, Patrick, International Law and The Rights of Minorities, 1991, S. 126f.; König / Peters, s. Fn 77, S. 1205.

vgl. Nowak, s. Fn 58, S. 593 f.

Siehe Fn 65.

96

Frowein, s. Fn 48, S. 77.

vgl. auch Giegerich, s. Fn 68, 2001, S. 241 ff., 268 f.

Frowein, s. Fn 48, S. 73 ff., 79.

99

vgl. Giegerich, s. Fn 68, S. 241ff, 269; vgl. auch das Urteil des EGMR Ždanoka gegen Litauen Nr. 58278/00 vom 16 März 2006, European Human Rights Report, Vol. 45 (2007) S. 478 ff.,501, in dem die Verhältnismäßigkeit eines Ausschlusses vom passiven Wahlrecht zum Schutz der verfassungsmäßigen Ordnung ohne individuelle Prüfung abgelehnt wurde, soweit keine besonderen und außergewöhnlichen Rechtfertigungsgründe vorliegen. 
bereits unzulässig ist, die Anhänger einer bestimmten Religion als generell unzuverlässig und ungeeignet zur Gewährleistung der Aufrechterhaltung der verfassungsmäßigen Ordnung zu erklären, so ist es a fortiori unzulässig, wenn sich diese Vermutung, wie im vorliegenden Fall, auf die Anhänger aller Religionen mit Ausnahme der Staatsreligion bezieht. Eine Ideologie, ganz gleich ob es sich dabei um eine weltliche oder religiöse handelt, stellt einen Teil der nationalen Rechtsordnung dar, welche an die völkerrechtlichen Verpflichtungen eines Staates anzupassen ist, um nicht gegen Völkerrecht zu verstoßen (vgl. hierzu Artikel 27 der Wiener Vertragsrechts Konvention ${ }^{100}$ (WVK) und Artikel 2 Abs. 2 IPbpR).

Der Ausschluss nicht-muslimischer Staatsbürger von der Wählbarkeit zum Amt des Staatspräsidenten ist folglich mit dem völkerrechtlichen Verbot von Diskriminierungen nicht vereinbar.

Das allgemeine passive Wahlrecht

Das Wahlrecht in seiner aktiven wie in seiner passiven Ausgestaltung ist eines der wichtigsten politischen Rechte überhaupt. Das gleichberechtigte passive Wahlrecht aller Staatsbürger ist in verschiedenen regionalen wie internationalen Völkerrechtinstrumenten garantiert. Bereits in Artikel 21 Abs. 3 der Allgemeinen Erklärung der Menschenrechte wird festgestellt, dass der Wille des Volkes die Grundlage für die Autorität der öffentlichen Gewalt bildet und dass dieser Wille durch periodische und unverfälschte Wahlen mit allgemeinem und gleichem Wahlrecht bei geheimer Stimmabgabe oder in einem gleichwertigen Verfahren zum Ausdruck kommen muss. Afghanistan hat sich durch Artikel 7 AV innerstaatlich ausdrücklich zur Beachtung der Allgemeinen Erklärung der Menschenrechte verpflichtet. Das Wahlrecht und die Wählbarkeit aller Staatsbürger wird außerdem in Artikel 25 b) IPbpR des Internationalen Paktes für bürgerliche und politische Rechte garantiert. Ähnliche Bestimmungen finden sich auch in Artikel 23 b) der Amerikanischen Menschenrechtskonvention und in Artikel 13 der afrikanischen Banjul Charta der Menschenrechte und Rechte der Völker.

Wie bereits festgestellt wurde, ist das gleichberechtigte passive Wahlrecht aller Staatsbürger hinsichtlich aller jener Ämter anwendbar, welche nach der jeweiligen Verfassungsordnung durch Wahl zu besetzen sind. Dies beinhaltet die Verpflichtung des Staates, das Wahlrecht und die Wählbarkeit als Grundrecht aller Staatsbürger zu etablieren. Eine Begrenzung dieser Rechte auf bestimmte Gruppen oder Klassen ist dagegen unvereinbar mit diesem Grundsatz. ${ }^{101}$ Beschränkungen können zulässig sein, soweit diese nicht diskriminierend oder unangemessen sind. ${ }^{102}$ Wie allerdings bereits festgestellt wurde, stellt der

Vom 23. Mai 1969, in Kraft getreten am 27. Januar 1980; BGB1. 1985 II S. 926; UNTS Vol. 1155 S. 331.

\footnotetext{
Menschenrechtsausschuss, Einzelbeschwerde Nr. 500/1992, Joszef Debreczeny gegen Niederlande, Report of the Human Rights Committee, UN Doc. (A/50/40) Supplement No. 40, S. 59 ff., 64; Nowak, s. Fn 58, S. 576; ders. Politische Grundrechte, 1988, S. 329 ff.
} 
Ausschluss nicht-muslimischer Staatsbürger von der Wählbarkeit zum Präsidentenamt diesen gegenüber eine Diskriminierung dar. Dieser Ausschluss verstößt folglich auch gegen das völkerrechtlich garantierte passive Wahlrecht nicht-muslimischer Staatsbürger.

dd) Der Rang völkerrechtlicher Verträge in den beiden Rechtsordnungen

Da die Regelungen hinsichtlich der Wählbarkeit zum Staatspräsidenten gegen die völkerrechtlichen Verpflichtungen beider Staaten verstoßen, wie sie sich insbesondere aus dem IPbpR ergeben, stellt sich die Frage nach dem Rang völkerrechtlicher Verträge in den beiden nationalen Normenhierarchien. Artikel 9 des iranischen Zivilgesetzbuches ${ }^{103}$ sieht vor, dass völkerrechtliche Verträge, welche verfassungsgemäß ratifiziert wurden, einen integralen Bestandteil der iranischen Rechtsordnung darstellen und den Rang formeller Parlamentsgesetze besitzen. ${ }^{104}$ Zwar wurde die grundsätzliche unmittelbare Anwendbarkeit der Bestimmungen des IPbpR durch die iranische Justiz ausdrücklich bestätigt, ${ }^{105}$ als im Rang eines Gesetzes stehend sind diese in der nationalen Normenhierarchie aber der Verfassung und damit auch dem islamischem Recht untergeordnet. ${ }^{106}$

Ähnlich ist die Rechtslage in Afghanistan. Auch wenn die afghanische Verfassung in Artikel 6 die Bedeutung der Menschenrechte betont und den Staat in Artikel 7 AV ausdrücklich zur Beachtung der internationalen Verträge verpflichtet, ergibt sich daraus nicht zwingend der Verfassungsrang einzelner völkerrechtlicher Verträge. Eindeutige Regelungen zum Rangverhältnis zwischen Völkerrecht und Verfassung finden sich nicht in der afghanischen Verfassung. In der afghanischen Rechtswissenschaft geht man davon aus, dass völkerrechtlichen Verträgen der Rang von Parlamentsgesetzen zukommt. ${ }^{107}$ Diese Ansicht wird von Artikel 121 AV bestätigt, der als eine der Aufgaben des afghanischen

Badrian Fakhreddin, The Civil Code of Iran (qānun e madani) [Englisch-Persische Ausgabe], Teheran 1380 / 2001.

Vgl. dazu auch Harland, Christopher, The Status of the International Covenant on Civil and Political Rights (ICCPR) in the Domestic Law of State Parties, Human Rights Quarterly, Vol. 22 (2000), S. 187 ff., 225; Nowak, Fn 58, S. 59.

Gutachten Nr. 7/1669 der Rechtsabteilung der iranischen Justiz vom 19. Oktober 1992; vgl. zu diesem auch Consideration of Reports Submitted by States Parties Under Artikel 40 of the Covenant: Iran, UN GAOR, Human Rights Comission, 46 ${ }^{\text {th }}$ Session, 1193 Meeting; UN Doc. CCPR/C/SR. 1193 (1992), S. 15; Harland, s. Fn 104, S. 187 ff., 225.

Vgl. auch die Aussage des iranischen Delegierten Khosroshahi vor dem Menschenrechtsausschuss "He [d. h. der iranische Delegierte Anm. d. Verf.] felt bound to emphasize, that although many articles of the Covenant [d. h. der IPbpR Anm. d. Verf.] were in conformity with the teachings of Islam, there could be no doubt that the tenets of Islam would prevail whenever the two sets of laws were in conflict." Zusammenfassung des Staatenberichts gemäß Artikel $40 \mathrm{IPbpR}$ der I. R. Iran vom 15. Juli 1982, UN Doc. CCPR/C/SR. 364 (1982), S. 3 § 4

\footnotetext{
So das Ergebnis der Recherchen von Frau Mandana Knust Rasekh Afsah, Wissenschaftliche Mitarbeiterin im Afghanistan Projekt des Max-Planck-Institutes für ausländisches öffentliches Recht und Völkerrecht, der an dieser Stelle herzlich gedankt sei.
} 
Obersten Gerichtshofs die Überprüfung internationaler Vereinbarungen auf ihre Übereinstimmung mit der Verfassung nennt. Hieraus lässt sich folgern, dass völkerrechtliche Verträge im Rang unter den Bestimmungen der Verfassung stehen. Damit gilt für beide Staaten, dass die Völkerrechtswidrigkeit der untersuchten Bestimmungen keine Wirkung für deren innerstaatliche Wirksamkeit hat. Denn die hier aufgezeigten Ungleichbehandlungen sind in beiden Staaten in den Verfassungen selbst vorgesehen, welche in den jeweiligen nationalen Normenhierarchien einen höheren Rang bekleiden als die Bestimmungen völkerrechtlicher Verträge.

Die Situation stellt sich aus völkerrechtlicher Perspektive allerdings insoweit anders dar, als im Völkerrecht zwar keine Regel dahingehend existiert, dass völkerrechtswidriges nationales Recht unwirksam wäre. ${ }^{108}$ Aber da zumindest bezüglich des IPbpR keiner der beiden Staaten einen Vorbehalt eingelegt hat, sind beide verpflichtet, entgegenstehende Bestimmungen ihrer nationalen Rechtsordnungen an dessen Regelungen anzupassen. Es ist allgemein anerkannt, dass völkerrechtliche Verpflichtungen insofern in der Normenhierarchie über dem nationalen Recht stehen, als der Staat verpflichtet ist, sein innerstaatliches Recht völkerrechtskonform auszugestalten. ${ }^{109}$, ${ }^{110}$ Der Staat kann sich deshalb nicht auf dem Völkerrecht widersprechendes nationales Recht berufen, um sich seiner völkerrechtlichen Verantwortlichkeit zu entziehen, ganz gleich, welchen Rang die völkerrechtswidrigen Bestimmungen innerstaatlich haben. ${ }^{11}$ Hinsichtlich der Rechte aus dem IPbpR ergibt sich der Vorrang des Völkerrechts auch aus Artikel 2 Abs. 2 IPbpR. Darum lässt auch die Behauptung eines Staates, auf Grund von besonderen verfassungsrechtlichen Voraussetzungen die notwendigen Anpassungen seiner Rechtsordnung nicht leisten zu können, seine

108

Dahm, Georg / Delbrück, Jost / Wolfrum, Rüdiger, Völkerrecht, Band I/1, Berlin, 1988, S. 102 f.; Brownlie Ian, Public International Law, Oxford, 2003, S. 34.

109 Dahm / Delbrück / Wolfrum, s. Fn 108, S. 102; Partsch, Karl Josef, International Law and Municipal Law, in: Bernhardt, Rudolf (Hrsg.), Max-Planck-Institut für Ausländisches Öffentliches Recht und Völkerrecht, Encyclopedia of public international law (EPIL), Band II 2, Amsterdam, 1992, S. 1183 ff., 1190; vgl. auch Brownlie, s. Fn 108, S. 34f.

110

Diese Verpflichtung hat bereits der Ständige Internationale Gerichtshofs in seinem Gutachten zum Bevölkerungsaustausch zwischen Griechenland und der Türkei erkannt und bestätigt. Advisory Opinion, Exchange of Greek and Turkish Population, vom 21. Februar 1925, PCIJ Series B No. 10, S. 20.

111

Dahm / Delbrück / Wolfrum, s. Fn 108, S. 103; Brownlie, s. Fn 108, S. 34f.; Partsch, s. Fn 108, S. 1183ff., 1190; Oppenheim, Lassa / Jennings, Robert / Watts, Arthur, International Law, Harlow, 1992, Vol I/1, S. 84 m. w. N. Dieser Grundsatz wurde bereits im Alabama Schiedsspruch aus dem Jahre 1872 anerkannt und seitdem in ständiger Praxis von internationalen Gerichten bestätigt. Moore, John Bassett, History and digest of the international arbitrations to which the United States has been a party, Washington, 1898, S. 653 ff., 659; Advisory Opinion, Greco-Bulgarian Communities Case, vom 31. Juli 1930 PCIJ Series B No. 17, S. 32; ICJ Reports 1955, Nottebohm Case, vom 6. April 1955, S. 20 f.; vgl. auch Brownlie, s. Fn 108, S. 34 f. Dieser völkergewohnheitsrechtliche Grundsatz ist auch in Artikel 27 der Wiener Vertragsrechtskonvention verankert. 
völkerrechtlichen Verpflichtungen unberührt. ${ }^{112}$ Völkerrechtlich sind daher beide Staaten verpflichtet, ihre Verfassungen an die international garantierten Menschenrechte anzupassen. Im Falle Afghanistans kommt hinzu, dass die afghanische Verfassung den Staat in Artikel 7 ausdrücklich zur Beachtung der völkerrechtlichen Verträge und der Allgemeinen Erklärung der Menschenrechte verpflichtet.

\section{d) Zwischenergebnis}

Aus der Verankerung des Staatsprinzips einer islamischen Republik in der Verfassungsordnung ergibt sich, dass bestimmte Staatsämter, wie etwa jenes des Staatspräsidenten, Muslimen vorbehalten sind. Derartige Ungleichbehandlungen zwischen muslimischen und nichtmuslimischen Staatsbürgern verstoßen allerdings sowohl gegen die völkerrechtlich geschützte negative Religionsfreiheit nicht-muslimischer Staatsbürger und gegen ihr völkerrechtlich garantiertes gleichberechtigtes Wahlrecht als auch gegen das Verbot von Diskriminierungen. Auch wenn dies innerstaatlich nichts an der Wirksamkeit der betreffenden Regelungen ändert, trifft beide Staaten doch die völkerrechtliche Verpflichtung, diese Rechtsverstöße zu beseitigen.

Nachdem damit Auswirkungen des Staatsprinzips identifiziert wurden, welche per se im Konflikt zu den Menschenrechten stehen, werden im Folgenden die Auswirkungen der Etablierung einer islamischen Republik herausgearbeitet, welche konstitutiv für eine solche sind und zwar selbst keine unmittelbaren Verstöße gegen Menschenrechte beinhalten, aber je nach Auslegung des islamischen Rechts zu solchen führen können.

\section{Für die Menschenrechte potentiell problematische Auswirkungen aus dem Staatsprinzip einer islamischen Republik}

$\mathrm{Zu}$ den Auswirkungen aus dem Staatsprinzip einer islamischen Republik, die potentiell zu Verstößen gegen die Menschenrechte führen können, gehören jene Bestimmungen, welche erstens den Islam beziehungsweise einer seiner Rechtsschulen als Staatsreligion verankern und zweitens die Übereinstimmung der Rechtsordnung mit dem islamischen Recht einer bestimmten Rechtsschule oder zumindest mit Teilen davon erfordern.

Die Ableitung dieser Regelungen aus dem Prinzip der Islamischen Republik ergibt sich daraus, dass nach einhelliger Ansicht der Befürworter eines islamischen Staates die Orientierung der staatlichen Rechtsordnung an der Šarî'a und damit verbunden die Einführung des Islams als Staatsreligion konstitutiv für einen solchen sind. Nach deren Ansicht kann sich ein Staat nur dann als islamisch bezeichnen, wenn er auf der $\check{S} a r \hat{\imath} ' a$ beruht und diese

112

Oppenheim / Jennings / Watts, s. Fn 111, S. 85; vgl. auch Ständiger Internationaler Gerichtshof, Advisory Opinion, Treatment of Polish Nationals and other Persons of Polish origin and speech in the Danzig Territory, vom 04. Februar 1932, PCIJ Series A/B No. 44, S. 24; Georges Pinson Fall, in: McNair, Arnold D. / Lauterpacht, Hersch, Annual Digest of Public International Law Cases, London, Vol 4 (1927 - 1928), S. 9 ff., 10 f. 
auch durchgesetzt wird. ${ }^{113}$ Bestimmungen, welche sich auf diese Forderung zurückführen lassen und eine Übereinstimmung der nationalen Rechtsordnung mit der Šarî'a verlangen, finden sich daher in den Rechtsordnungen aller islamischen Republiken. ${ }^{114}$ In diesem Zusammenhang sind in den jeweiligen Rechtsordnungen außerdem regelmäßig Verfahren der Kontrolle der Gesetze auf ihre Übereinstimmung mit dem islamischen Recht beziehungsweise mit der Šarî'a vorgesehen. ${ }^{11}$

Wie noch zu zeigen sein wird, kann der Grad der geforderten Übereinstimmung der Rechtsordnung mit dem islamischen Recht dabei in den Verfassungsordnungen der verschiedenen islamischen Republiken durchaus unterschiedlich ausgestaltet sein. Es kann sowohl eine Übereinstimmung nur mit der $\breve{S} a r \hat{\imath} ’ a$ gefordert sein, als auch mit dem gesamten islamischen Recht einer bestimmten Rechtsschule des Islams. Dies bedeutet, dass diese Rechtsordnung nicht nur mit der Šarî'a übereinstimmen muss, sondern auch mit dem fiqh der betreffenden Rechtsschule. Als fiqh werden die Regelungen bezeichnet, welche von den Rechtsgelehrten einer bestimmten Rechtsschule des Islams aus der Šarî'a abgeleitet werden. Fiqh wird beschrieben als ,das Wissen von den gesetzlichen Bestimmungen für das Recht der Einzelfälle, abgeleitet aus den klar verständlichen Rechtsquellen [d.h. Koran und Sunna ${ }^{116 ، “ .117}$ Die Wissenschaft des fiqh beschäftigt sich mit der Anwendung der Šarî'a auf das alltägliche Leben und der Ableitung differenzierter Handlungsanleitungen aus den göttlichen Geboten. Da in der Šarî'a nach islamischer Dogmatik ein allumfassendes Rechtssystem zu sehen ist, welches allerdings der Auslegung und Präzisierung bedarf, zielt die islamische Rechtswissenschaft darauf ab, jeden Aspekt des Lebens auf Grundlage der Šarî'a zu bewerten und zu regeln. ${ }^{118}$ Die Vorgaben, welche sich aus der Kombination aus Šarî'a und fiqh für eine Rechtsordnung ergeben, sind daher sehr viel enger als jene, welche alleine aus der Šarî'a folgen.

a) Iran

Die iranische Verfassung bestimmt durch Artikel 12 IrV den Islam in der Interpretation der zwölferschiitischen ğafari Rechtsschule zur ewigen Staatsreligion des Landes.

Khomeini, s. Fn 34, S. 40 ff.; vgl. auch Maududi, s. Fn 24, S. 45 ff.; Asad, s. Fn 12, S. 34.; vgl. Seyyid Qutb, Milestones, Damaskus, S. 9.

Vgl. für das Gebot der Übereinstimmung der Rechtsordnung mit dem islamischen Rechts beziehungsweise der Šarî'a Artikel 3 AV, Artikel 4 IrV, Artikel 227 Abs. 1 der pakistanischen Verfassung, vgl. auch die Präambel der mauretanischen Verfassung, welche vorsieht, dass die Gebote des Islams die einzige Quelle der Gesetzgebung darstellen.

115 Auch wenn die Etablierung eines solchen Verfahrens nicht konstitutiv für eine islamische Republik ist, wie das Beispiel Mauretaniens zeigt, dessen Verfassungsordnung ein solches Verfahren nicht kennt.

116 Anmerkung des Autors.

117 Löschner, s. Fn 18, S. 27; El Baradie, s. Fn 17, S. 43.

118

Abdal-Haqq, s. Fn 17, S. 5. 
Artikel 4 IrV legt fest, dass alle zivilrechtlichen, strafrechtlichen, finanziellen, ökonomischen, verwaltungsrechtlichen, kulturellen, militärischen, politischen und sonstigen Gesetze und Vorschriften auf den „Regelungen des Islams“ (mavāzin e eslāmi) beruhen müssen. Diese Regelung bestimmt sowohl den Inhalt und Umfang aller einfachen Gesetze und Vorschriften des Landes als auch den der Verfassung selbst.

Mit der Kontrolle der Implementierung des Artikels $4 \mathrm{IrV}$ sind nach den Artikeln 4, 72 und $96 \mathrm{IrV}$ die Rechtsgelehrten des so genannten Wächterrates (shurā ye negahbān) betraut. Bei diesem Rat handelt es sich nach Artikel 91 IrV um ein Gremium aus insgesamt zwölf Mitgliedern, welches sich aus sechs weltlichen Juristen und sechs Gelehrten des Rechts der schiitischen ğafari Rechtsschule zusammensetzt. Für die Kontrolle der Konformität der Rechtsordnung mit dem islamischen Recht sind allerdings alleine letztere zuständig. Zentrale Aufgabe des Wächterrats ist nach Artikel 94 der iranischen Verfassung die präventive Prüfung der Übereinstimmung der Parlamentsgesetze mit der Verfassung und mit den Geboten des Islams. Zur Erfüllung dieser Aufgabe sind sämtliche Gesetzesbeschlüsse des Parlaments, bevor diese in Kraft treten können, zunächst dem Wächterrat zur Prüfung vorzulegen. Nur wenn dieser ihre Übereinstimmung sowohl mit der Verfassung als auch mit den Geboten des Islams positiv feststellt, werden diese wirksam, andernfalls sind sie dem Parlament zur erneuten Beratung zurückzuleiten. ${ }^{119}$ Als Konsequenz der obligatorischen Prüfung der Gesetzesvorlagen durch den Wächterrat fehlen dem Parlament jegliche legislativen Befugnisse, solange der Wächterrat nicht funktionsfähig ist. ${ }^{120}$ Der Wächterrat sieht seine Befugnis zur Verwerfung von Gesetzen allerdings nicht als auf die ihm vorgelegten und noch nicht in Kraft getretenen Gesetze beschränkt an. Er sieht sich vielmehr auch als befugt, bereits in Kraft getretene Gesetze auf ihre Übereinstimmung mit der Verfassung und den Geboten des Islams hin zu überprüfen. ${ }^{121} \mathrm{Da}$ der Wächterrat nach Artikel 98 der Verfassung als einziges Verfassungsorgan zur verbindlichen Auslegung der Verfassung befugt ist, ${ }^{122}$ wird diese Interpretation des Wächterrates auch nahezu allgemein akzeptiert.

Der Begriff der „Regelungen des Islams“ in Artikel 4 IrV bezieht sich dabei auf das gesamte islamische Recht der ğafari Rechtsschule und beschränkt sich folglich nicht auf

Artikel 94, 96 IrV

20 Artikel 93 IrV; Ausnahmen von diesem Grundsatz bilden lediglich die Wahl der sechs weltlichen Juristen des Wächterrates durch das Parlament sowie die Entgegennahme der Beglaubigungsschreiben der Abgeordneten zu Beginn der Legislaturperiode, vgl. Artikel 93 IrV.

121 Ansicht des Wächterrates Nr. 1983 vom 08.02.1360 (1981) zu finden in Hāshemi,, s. Fn 87, S. 242. Der Wächterrat beschränkt diese Kompetenz dabei nicht nur auf vor-konstitutionelle Gesetze.

Eine Interpretation der Verfassung durch den Wächterrat ist wirksam, wenn diese mit einer Mehrheit von Dreivierteln seiner Mitglieder beschlossen wird. 
die Šarî'a, sondern schließt auch den fiqh ein. ${ }^{123}$ Zwar erscheint der Wortlaut der Vorschrift in dieser Hinsicht unklar. Allerdings sah man bei den Verhandlungen zu Artikel 4 der Verfassung davon ab, den Begriff der „Regelungen des Islams“ durch den Zusatz, wie sie in „Koran und Sunna bestimmt sind“ auf die Šarî'a einzuschränken, obwohl dies wiederholt vorgeschlagen wurde. ${ }^{124}$ Auch wenn keine Begründung dafür angegeben wurde, warum dieser Zusatz nicht aufgenommen wurde, spricht dies doch dafür, dass sich dieser Begriff nicht auf die $\breve{S} a r \hat{\imath}^{\prime} a$ beschränkt. ${ }^{125}$ Dies wird durch die Praxis des Wächterrates bestätigt, der den Begriff sowohl auf die Šarî'a als auch auf den fiqh bezieht. ${ }^{126}$ Der Praxis des Wächterrates kommt deshalb besonderes Gewicht zu, da diesem nicht nur die Kontrolle der Übereinstimmung der Rechtsordnung mit den „Regelungen des Islams“ obliegt, sondern, wie bereits erwähnt wurde, nach Artikel 98 IrV als einzigem Verfassungsorgan auch die Auslegung der Verfassung selbst. Aus Artikel $72 \mathrm{IrV}$ in Verbindung mit Artikel $12 \mathrm{IrV}$ ergibt sich außerdem, dass sich die „Regelungen des Islams“ in Artikel 4 IrV ausschließlich auf die $\breve{S} a r \hat{\imath}^{\prime} a$ und den fiqh in der Auslegung der ğafari Rechtsschule beziehen.

\section{b) Afghanistan}

Auch die afghanische Verfassung etabliert in Artikel 2 AV den Islam als Staatsreligion. Zu betonen ist, dass dabei im Gegensatz zu früheren afghanischen Verfassungen allerdings keine Festlegung auf eine bestimmte Rechtsschule des Islams erfolgt. ${ }^{127}$

Artikel 3 AV bestimmt, dass in Afghanistan kein Gesetz dem Glauben und den Bestimmungen der heiligen Religion des Islam widersprechen darf. Wie sich aus Artikel $121 \mathrm{AV}$ in Verbindung mit Artikel 3 AV ergibt, ist der Oberste Gerichtshof Afghanistans auf Antrag eines Gerichts oder der Regierung zur Kontrolle darüber befugt, ob die Gesetze die Vorgaben des Artikels 3 AV beachten.

Fraglich ist allerdings, ob Artikels 3 AV eine Übereinstimmung der Rechtsordnung auch mit dem fiqh einer bestimmten Rechtsschule verlangt, wenn er vorsieht, dass ,kein

So auch Mahmoudi Said, The Sharî'a in the New Afghan Constitution, Zeitschrift für ausländisches öffentliches Recht und Völkerrecht, Vol. 64 (2004), S. 867 ff., 871; Dr. Hassan Rezzai, wissenschaftlicher Mitarbeiter des Max-Planck-Institut für ausländisches und internationals Strafrecht, im Interview am 06. Februar 2008.

Surat e mashruh e mozākerāt e madjles e barresi nehā'i je qānun e asāsi je djomhuri je eslāmi je irān (Protokolle der Versammlung zur endgültigen Überprüfung der Verfassung der Islamischen Republik Iran), Band I, Teheran 1364 (1985), S. 313 ff.

Surat e mashruh, s.o.

Mahmoudi, s. Fn 123, S. 867 ff., 871; Dr. Hassan Rezzai, wissenschaftlicher Mitarbeiter des MaxPlanck-Institut für ausländisches und internationals Strafrecht, im Interview am 06. Februar 2008.

Vgl. Artikel 2 der Verfassung von 1964, welcher vorsah, dass für religiöse Handlungen und Zere128 monien des Staates die Hanafi Rechtsschule verbindlich sein sollte.

Grote, Rainer, Separation of Powers in the New Afghan Constitution, ZaöRV Vol. 64 (2004), S. 897 ff., 912; vgl. auch Mahmoudi, s. Fn 123, S. 867 ff., 87. 
Gesetz dem Glauben und den Bestimmungen der heiligen Religion des Islam widersprechen darf“. ${ }^{129}$ Aus dem Staatsprinzip der islamischen Republik ergibt sich nach dem oben gesagten nur, dass die Rechtsordnung des Staates mit der Šarî'a übereinstimmen muss, nicht aber notwendigerweise auch mit den Regelungen des fiqh einer bestimmten Rechtsschule. Als Beispiel hierfür kann auf die pakistanische Verfassung verwiesen werden, in welche den Vorbehalt des islamischen Rechts ausdrücklich nur auf die Šarî'a im eigentlichen Sinne beschränkt, wenn sie in Artikel 227 Abs. 1 festlegt, dass alle Gesetze in Übereinstimmung mit „den Geboten des Islams gebracht werden müssen, so wie sie im heiligen Koran und der Sunna festgelegt sind“, was als Umschreibung für die Šarî'a zu lesen ist.

Für eine Beschränkung des Artikels 3 AV auf die Šarî'a spricht, dass sich die afghanische Verfassung auf keine bestimmte Rechtsschule des Islams als Staatsreligion festlegt. Im Falle, dass sich die „Bestimmungen des Islams“ in Artikel 3 AV auch auf die Regelungen des fiqh beziehen sollten, wäre deshalb unklar, der fiqh welcher Rechtsschule gemeint sein sollte. Zwar genießt die Hanafi Rechtsschule, welche traditionell die dominante Rechtsschule Afghanistans ist, nach Artikel 130 AV insoweit eine hervorgehobene Stellung, als den Regelungen des fiqh dieser Rechtsschule für die Rechtssprechung eine subsidiäre Bedeutung zukommt im Falle, dass in der Verfassung und den sonstigen Gesetzen keine Bestimmungen zur Lösung eines Rechtsstreites zu finden sind. Daraus kann aber nicht geschlossen werden, dass Artikel 3 AV den fiqh dieser Rechtsschule in die „Bestimmungen des Islams“ mit einbezieht, ist es doch ein wesentlicher Unterschied, ob dem fiqh eine subsidiäre Rolle für die Urteilsfindung in einem Land zuerkannt wird, in dem für viele Bereiche noch keine gesetzlichen Kodifizierungen vorhanden sind, oder ob sich die gesamte Rechtsordnung nach dessen Vorgaben richten muss. Schließlich spricht auch die Qualifikation der Richter des Obersten Gerichtshofes als der Instanz, welcher die Kontrolle der Implementierung des Artikels 3 AV obliegt, dafür, dass der Begriff der „Bestimmungen des Islams“ nur die Šarî'a selbst umfasst. Artikel 118 Nr. 3 AV bestimmt, dass ein Kandidat für einen der Richterposten am Obersten Gerichtshof entweder über einen Hochschulabschluss der (weltlichen) Rechtswissenschaft oder der islamischen Rechtswissenschaft verfügen muss. Zwar ist es nach Artikel 118 Nr. 3 AV erforderlich, dass Kandidaten über ausreichende Kompetenz und Berufserfahrung im afghanischen Rechtssystem verfügen müssen, woraus sich ergibt, dass Kandidaten sowohl Erfahrungen in Bezug auf die geschriebene staatliche Rechtsordnung aufweisen müssen als auch hinsichtlich der Grundprinzipien des religiösen Rechts, weil das afghanische Rechtssystem sowohl durch moderne Gesetzeskodifikationen als auch durch religiöses Recht geprägt wird. Aus diesen Bestimmungen ergeben sich aber anders als aus den entsprechenden Regelungen der iranischen Verfassung keine Vorgaben dahingehend, dass eine bestimmte Anzahl von Richtern Experten des fiqh einer bestimmten Rechtsschule sein müssten. Von den gesetzlichen Vorgaben her ist es damit nicht garantiert, dass dem Obersten Gerichtshof Richter angehören, welche über detaillierte Kenntnisse des fiqh einer bestimmten Rechtsschule verfügen. Da 
durch die gesetzlichen Vorgaben damit nicht garantiert wird, dass dem Obersten Gerichtshof überhaupt die erforderliche Sachkompetenz zukommt, detailliert über die Vereinbarkeit eines Gesetzes mit dem fiqh einer bestimmten Rechtsschule zu entscheiden, spricht dies dafür, dass sich auch der Prüfungsmaßstab, welchen der Oberste Gerichtshof im Rahmen des Artikels 3 AV anwendet, nicht auf den fiqh einer bestimmten Rechtsschule bezieht.

\section{c) Das Verhältnis dieser Regelungen zu den Menschenrechten}

Diese dritte Kategorie von Regelungen, welche aus der Etablierung des Staatsprinzips folgt, beinhalten zwar selbst unmittelbar keinen Verstoß gegen Menschenrechte, ein solcher kann sich aber mittelbar aus diesen ergeben, beispielsweise aus dem Gebot der Übereinstimmung der nationalen Rechtsordnung mit dem islamischen Recht beziehungsweise der Šarî'a. So stellt etwa die Festlegung einer Staatsreligion für sich genommen grundsätzlich noch keinen Verstoß gegen die Religionsfreiheit der Anhänger anderer religiöser Bekenntnisse dar. ${ }^{130}$ Dies zeigt sich auch daran, dass sowohl der Menschenrechtsausschuss der Vereinten Nationen ${ }^{131}$ als auch die Europäische Kommission für Menschenrechte ${ }^{132}$ und die verschiedenen Sonderberichterstatter der Vereinten Nationen über religiöse Intoleranz ${ }^{133}$ explizit davon ausgehen, dass es für die Anwendung der Religionsfreiheit keinen Unterschied macht, welchen Rahmen eine nationale Rechtsordnung zur institutionellen Ausgestaltung der Beziehung zwischen Staat und Religion wählt. Ein Verstoß gegen die Religionsfreiheit Andersgläubiger ergibt sich aber dann, wenn, wie im Falle Irans und Afghanistan, aus dem islamischen Recht abgeleitet wird, dass ausschließlich die Anhänger der Staatsreligion Zugang zu bestimmten Staatsämtern haben.

Das Gebot der Übereinstimmung der nationalen Rechtsordnung mit dem islamischen Recht beziehungsweise mit der $\check{S}^{\prime} a \hat{i ̂}^{\prime} a$ ist deshalb nur dann mit den völkerrechtlich geschützten Menschenrechten vereinbar, wenn dieses Gebot insoweit relativiert wird, als die Verbindlichkeit des islamischen Rechts nur noch dann eingreift, wenn dessen Vorgaben ihrerseits mit den Menschenrechten vereinbar sind. Damit könnten in einer islamischen Republik einerseits die oben dargestellten Ziele sozialer Gerechtigkeit verfolgt werden,

Nowak, s. Fn 58, S. 415; Frowein, Jochen Abr., Religion and Religious Symbols in European and International Law, in: Brugger, Winfried / Karayanni, Michael (Hrsg.), Religion in the Public Sphere: A Comparative Analysis of German, Israeli, American and International Law, Berlin, 2007, S. 243 ff., 245.; ders., s. Fn 48, S. 73 ff., 78; Walter, Christian, Religionsverfassungsrecht, 2006 S. 392; Zur diesbezüglichen Rechtsprechung des EGMR ders. in: Grote, Rainer / Marauhn, Thilo (Hrsg.), EMRK/GG Konkordanzkommentar, Tübingen, 2006, S. 826; Evans, Carolyn, Freedom of Religion under the European Convention on Human Rights, Oxford, 2001, S. 80.

Menschenrechtsausschuss, General Comment Nr. 22, s. Fn 58, § 9 f.

132 Europäische Menschenrechtskommission, Darby gegen Sweden, Series A, Vol. 187, § 45, S. 17. 
welche dieses Verfassungsmodell gerade attraktiv machen. Auch wären durch das islamische Recht bedingte Beschränkungen der Menschenrechte wie etwa Einschränkungen der Meinungsfreiheit hinsichtlich als blasphemisch aufgefasster Meinungsäußerungen möglich, solange diese ihrerseits völkerrechtskonform sind. Andererseits wäre es aber nicht mehr länger möglich die Diskriminierung Andersgläubiger oder die Verhängung drakonischer Strafen unter Berufung auf das islamische Recht zu rechtfertigen. Diese Uminterpretation des Islamvorbehalts könnte durch eine Neuinterpretation des islamischen Rechts unterstützt werden, welche sich ausdrücklich an der Verwirklichung der Menschenrechte orientiert. ${ }^{134}$ Bei der Analyse und Bewertung der Unterschiede der beiden hier untersuchten Verfassungen wird der Schwerpunkt daher auf der Frage liegen, inwieweit diese derartigen Neuinterpretationen des islamischen Rechts Raum lassen.

\section{Die Unterschiede beider Verfassungsordnungen}

\section{a) Die Regelungen der ersten beiden Kategorien}

Im Hinblick auf die Reglungen der ersten Kategorie zeichnet sich die iranische Verfassung gegenüber der afghanischen durch eine weit stärkere Betonung sozialer Gerechtigkeit aus, insbesondere werden zum Teil sehr detaillierte Vorgaben für das nach dieser Verfassung zu errichtende Wirtschaftssystem gemacht. Dies erklärt sich aus dem großen Einfluss linker und links-islamischer Gruppierungen während der Revolution von 1979 und den anschlieBenden Verfassungsberatungen.

Bezüglich der problematischen Regelungen der zweiten Kategorie sind die Bestimmungen der iranischen Verfassung deutlich negativer zu beurteilen als jene der afghanischen, denn nach der iranischen Verfassung sind nicht nur Nicht-Muslime von zahlreichen Staatsämtern ausgeschlossen, sondern in der Regel erstreckt sich dieser Ausschluss auch auf nicht-schiitische Muslime. Die immense praktische Relevanz dieser Diskriminierungen ergibt sich, wenn man sich vergegenwärtigt, dass der Anteil letzterer an der iranischen Bevölkerung bei ungefähr zehn Prozent angesiedelt wird. ${ }^{135}$

\section{b) Die Regelungen der dritten Kategorien und die mit der Kontrolle der Gesetze betrauten Verfassungsorgane}

Die Regelungen der dritten Kategorie betreffend weisen beide Verfassungsordnungen erhebliche Unterschiede auf. Der augenscheinlichste Unterschied bezieht sich auf die Staatsreligion, denn während die afghanische Verfassung den Islam an sich zur Staatsreli-

\footnotetext{
134 Vgl. zu so einem Ansatz etwa der iranische Reformtheologe Mohsen, Kadivar, s. Fn 38; Vgl. auch den Bericht über Mohsen Kadivar von Bahman Nirumand vom 19. Januar 2005, http://www. 135 qantara.de/webcom/show_article.php/_c-578/_nr-8/_p-1/i.html (Letzter Besuch 03. März 2008).

So Buchta Wilfried, s. Fn 18, S. 173 ders., Who Rules Iran-The Structure of Power in the Islamic Republic, Washington D.C., 2000, S. 105; Amor, s. Fn 32 Rn 28.
} 
gion erklärt, ohne sich dabei auf eine bestimmte Rechtsschule festzulegen, bestimmt die iranische Verfassung die gafaritische Rechtsschule des Islams zur Staatsreligion.

Außerdem ist der Spielraum, welchen der Islamvorbehalt der Gesetzgebung belässt, in der iranischen Verfassung wesentlich enger ausgestaltet als in der afghanischen. Denn in der I. R. Iran müssen nach Artikel 4 IrV alle Gesetze des Landes sowie die Verfassung selbst nicht nur auf der Šarî'a basieren, sondern auch auf den Regelungen des fiqh der ğafari Rechtsschule. Die entsprechende Bestimmung des Artikels 3 AV der afghanischen Verfassung bezieht sich dagegen nur auf die Šarî'a selbst. Dies verleiht der afghanischen Verfassung einen wesentlich größeren Spielraum, um auf Veränderungen und die Anforderungen der Moderne zu reagieren, sind doch die sich aus Šarî'a und dem fiqh für eine Rechtsordnung ergebenden Vorgaben sehr viel enger gefasst als jene, die sich alleine aus der $\breve{S} a r \hat{i}^{\prime} a$, also aus Koran und Sunna, ergeben. Für die Verwirklichung der Menschenrechte kann sich außerdem positiv auswirken, dass die afghanische Verfassung den Staat nicht nur zur Beachtung der Bestimmungen des Islams verpflichtet, sondern auch zum Schutz der Menschenrechte und der Menschenwürde. Die Bindung des afghanischen Staates an die Menschenrechte wird dadurch noch verstärkt, dass Art. 7 AV die Pflicht zur Beachtung des Völkerrechts insbesondere im Hinblick auf den Schutz der Menschenrechte ausdrücklich festhält. Diese in der Verfassung selbst verankerte Pflicht zur Beachtung der Menschenrechte könnte sich als Einfallstor erweisen, um auf das islamische Recht zurückzuführende Bestimmungen, welche im Hinblick auf die Menschenrechte problematisch sind, zu reformieren. In der iranischen Verfassung findet sich keine vergleichbare Bestimmung. Soweit dort von Menschenrechten die Rede ist, werden diese nur in den Grenzen des Islams gewährt. ${ }^{136}$ In der afghanischen Rechtsordnung erscheint es daher anders als in der iranischen möglich, im Konfliktfall eine Abwägung zwischen den Geboten des Islams und den Menschenrechten zu treffen, wobei entscheidend sein wird, ob in der Rechtspraxis die Abwägungsresistenz des jeweiligen Kernbestands der Menschenrechte anerkannt wird. ${ }^{137}$

Wesentliche Unterschiede bestehen zwischen beiden Verfassungen schließlich auch im Hinblick auf die jeweils mit der Kontrolle der Konformität der Gesetze mit den Geboten des Islams betrauten Instanzen und insbesondere deren Zusammensetzung.

In der I. R. Iran obliegt die Kontrolle der Gesetze auf ihre Übereinstimung mit den Geboten des Islams ausschließlich den islamischen Rechtsgelehrten des Wächterrates, welche präventiv jedes Gesetz auf seine Konformität mit Šarî'a und fiqh der ğafari Rechtsschule zu überprüfen haben. Die Rechtsgelehrten des Wächterrates werden gemäß Artikel 91 Nr. 1 IrV vom Revolutionsführer ernannt. Der Revolutionsführer selbst wird nach Artikel 107 Abs. 1, 111 Abs. 1 IrV nicht direkt vom Volk gewählt, sondern von der so genannten Expertenversammlung auf Lebenszeit ernannt. Die Expertenversammlung wird zwar ihrerseits vom Volk gewählt, allerdings kommen als Kandidaten von Gesetzes wegen

\footnotetext{
136 Vgl. Artikel 20 ff. IrV.

137 Vgl. hierzu Knust Rassekh Afshar, s. Fn 35, S. 591 ff.
} 
nur islamische Rechtsgelehrte in Betracht. ${ }^{138}$ Die Überwachung der Wahlen zum Expertenrat obliegt nach Artikel $99 \mathrm{IrV}$ dem Wächterrat in seiner Gesamtheit, also sowohl den islamischen Rechtsgelehrten als auch den weltlichen Juristen. Der Wächterrat kann im Rahmen seiner Überwachungstätigkeit jedem Kandidaten die Teilnahme an den Wahlen verwehren, wenn dieser nach Ansicht des Wächterrates nicht die zahlreichen zu einer Teilnahme an der Wahl notwendigen Kriterien erfüllt.

In Afghanistan dagegen ist nach Artikel 121 AV der Oberste Gerichtshof mit der nachträglichen Normenkontrolle beauftragt. Da dieser anders als der iranische Wächterrat nur auf Antrag eines Gerichts oder der Regierung tätig werden kann, nicht aber ex officio, ist sein Einfluss auf die Gesetzgebung bereits aus diesem Grunde sehr viel geringer als jener des Wächterrates in der I. R. Iran. Entscheidend für den Unterschied zwischen beiden Organen sind aber vor allem die Zusammensetzung des afghanischen Obersten Gerichtshofes und die Methode der Richterauswahl. Die Richter des Obersten Gerichtshofes können zwar eine Ausbildung im religiösen Recht aufweisen, um sich für dieses Amt zu qualifizieren, müssen dies aber keineswegs, da Artikel 118 Nr. 3 AV die Möglichkeiten einer religiösen oder weltlichen rechtswissenschaftlichen Ausbildung als gleichberechtigte Alternativen anerkennt. Schließlich werden die Richter des Obersten Gerichtshofes durch den direkt vom Volk gewählten Staatspräsidenten mit Zustimmung durch das ebenfalls in direkter und allgemeiner Wahl gewählte Unterhaus (Wolesi Jirga) ernannt. Eine entsprechende Parlamentsmehrheit vorausgesetzt, steht der Ernennung von Richtern, welche für eine menschenrechtskonforme Neuinterpretation des islamischen Rechts eintreten, damit grundsätzlich kein Hindernis im Wege, vermag sich der Mehrheitswille des Volkes doch direkt auf die Wahl des Staatspräsidenten sowie die Zusammensetzung des Unterhauses und dadurch auch auf deren Ernennungspraxis auszuwirken. Vollkommen anders stellt sich dagegen die Lage in der I. R. Iran dar, denn der Wille der Mehrheit des Volkes vermag dort kaum Einfluss auf die Auswahl der Rechtsgelehrten des Wächterrates zu nehmen. Selbst die bereits nur schwach ausgeprägten indirekten Einflussmöglichkeiten über die Wahl des Expertenrates werden dadurch noch weiter abgeschwächt, dass erstens nur islamische Rechtsgelehrte als Mitglieder des Expertenrates in Betracht kommen und zweitens der amtierende Wächterrat jeden Kandidaten von der Wahl zu diesem Gremium ausschließen kann. Ein grundlegender Wandel in der Auffassung der Rechtsgelehrten des Wächterrates zum islamischen Recht erscheint daher auch bei einen entsprechenden Parlamentsmehrheiten als Ausdruck des Mehrheitswillen des Volkes nur sehr schwer vorstellbar.

$\S 2$ des Gesetzes über die Wahlen und die Geschäftsordnung der Expertenversammlung zur Ausführung der Artikel 5, 107 und 108 der Verfassung der I. R. Iran (qānun e entekhābāt va ā'innāme ye dākheli ye mağles khebregān marbut be osul e 5 va 107 va 108 qānun e asāsi ye ğomhuri ye eslami irān) vom 10. 7. 1359 (1980), veröffentlicht im Offiziellen Anzeiger (ruznāme ye rasmi) Nr. 10580 vom 06.04.1360 (1981), aktuelle Version mit sämtlichen Änderungen in: mă̆mu'e ye qavānin va mogararāt marbut be shar va shardāri (Sammlung der Gesetzen und Vorschriften), Teheran, 1385 (2006), S. 50 ff.; vgl. auch Hāshemi, s. Fn 18, S. 117; ders., s. Fn 87, S. 44 ff. 


\section{Ergebnis}

Abschließend ist daher festzuhalten, dass sich eine islamische Republik in erster Linie durch die Verbindlichkeit der Šarî'a, wenn nicht sogar des gesamten islamischen Rechts in der nationalen Rechtsordnung sowie durch die Etablierung des Islams als Staatsreligion von anderen Staaten unterscheidet. Auch wenn das Völkerrecht dadurch, dass es die Einrichtung einer Staatsreligion akzeptiert, mittelbar zum Ausdruck bringt, dass es die Gleichheit der Religionsgemeinschaften nicht garantiert, darf sich aber aus dem privilegierten Status einer Staatsreligion keine Diskriminierung der einzelnen Anhänger einer anderen Religion und keine Verletzung ihrer Freiheitsrechte entwickeln. Auch wenn die Etablierung einer islamischen Republik durchaus positive Auswirkungen hat, wie etwa die Bedeutung, welche wirtschaftlichen, sozialen und kulturellen Rechten beigemessen wird, ist es doch völkerrechtlich hoch problematisch, dass durch das Erfordernis der Übereinstimmung der nationalen Rechtsordnungen mit der $\breve{S} a \hat{r}^{\prime} a$ beziehungsweise mit dem gesamten Regelwerk einer bestimmten islamischen Rechtsschule, Diskriminierungen und Freiheitsrechtsverletzungen in diese inkorporiert werden. Hier kann nur eine grundlegende Neuninterpretation des islamischen Rechts wirkliche Abhilfe schaffen.

Trotz dieser problematischen Aspekte des Staatsprinzips muss hinsichtlich der Frage, ob die Vorbehalte begründet sind, welche der Errichtung einer islamischen Republik im Westen entgegengebracht werden, differenziert werden. Denn auch in diesen problematischen Punkten bestehen erhebliche Unterschiede zwischen den Verfassungen der verschiedenen islamischen Republiken, was sich gerade im Vergleich der Verfassungen Afghanistans und Irans zeigt. Die Verfassung der I. R. Afghanistan ist nicht nur sehr viel offener im Hinblick auf die Bedeutung des Islams für die Gesetzgebung formuliert als die iranische, sie enthält daneben vor allem eine ausdrückliche Verpflichtung zur Beachtung der völkerrechtlich garantierten Menschenrechte. Diese Bedeutung der Menschenrechte und des Völkerrechts in der Verfassung ist außergewöhnlich für die Region und könnte sich als eine Möglichkeit erweisen, im Falle des Konflikts zwischen völkerrechtlich garantierten Menschenrechten und islamischem Recht, den Kernbestand ersterer zu wahren. Hinzu kommt, dass die afghanische Verfassung einer der Verwirklichung der Menschenrechte verpflichtete Neuinterpretation des islamischen Rechts sehr viel mehr Spielraum lässt als die iranische, falls denn die afghanische Gesellschaft für eine solche Neuinterpretation eines Tages bereit sein sollte. Der Raum für eine Anpassung der Rechtsordnung an den gesellschaftlichen Wandel in der iranischen Verfassung ist dagegen sehr stark eingeschränkt. Da sich unter dem Mantel einer islamische Republik daher sehr verschiedene Verfassungsmodelle verbergen können, lässt sich die anfangs gestellte Frage, ob die Befürchtungen, welche diesem Staatsmodel im Westen entgegengebracht werden, berechtigt sind oder nicht, pauschal nicht beantworten. So ist die Verfassung der I. R. Afghanistan für die Region als ausgesprochen positiv und modern zu bewerten. Ihre Vorbildwirkung auf andere Staaten der Region wäre daher von einer menschenrechtlichen Perspektive zu begrüßen. Entscheidend für die Verwirklichung der Menschenrechte oder den Verstoß gegen diese sind daher 
in erster Linie die tatsächlichen Vorgaben der Verfassung im Spannungsfeld zwischen Islam und Menschenrechten und weniger die Frage, ob eine Verfassung die Staatsform einer islamischen Republik wählt oder nicht. 